\title{
Moving Algebraic Cycles of Bounded Degree
}

\author{
Eric M. Friedlander* and H. Blaine Lawson**
}

The Chow Moving Lemma is a theorem which asserts that a given algebraic $s$-cycle on a smooth algebraic variety $X$ can be moved within its rational equivalence class to intersect properly a given $r$-cycle on $X$ provided that $r+s \geq \operatorname{dim}(X)$ (cf. [Chow], [S2]). In the past few years, there has been considerable interest in studying spaces of algebraic cycles rather than simply cycles modulo an equivalence relation. With this in mind, it is natural to ask whether one can move a given "bounded family" of $s$-cycles on the smooth variety $X$ to intersect properly a given "bounded family" of $r$-cycles. The main point of this paper is to formulate and prove just such a result. In Theorem 3.1, we demonstrate that for any integer $e$ and any smooth projective variety $X$, one can simultaniously and algebraically "move" all effective $s$-cycles of degree $\leq e$ on $X$ so that each such cycle meets every effective $r$-cycle of degree $\leq e$ on $X$ in proper dimension.

The primary motivation for this Moving Lemma for Cycles of Bounded Degree was the possibility of a duality theorem between cohomology and homology theories defined in terms of homotopy groups of cycle spaces. Using Theorem 3.1, we have proved such a duality theorem for complex quasi-projective varieties in [F-L2]. We prove our Moving Lemma for varieties over an arbitary infinite field, permitting a proof in $[\mathrm{F}-\mathrm{V}]$ of a duality theorem for "motivic cohomology and homology".

The reader will find that our Moving Lemma has numerous good properties. First of all, the move is given as an algebraic move (parametrized by a punctured projective line) on Chow varietes. Although this move is "good" only for $s$-cycles of bounded degree, it is defined on all effective $s$-cycles. Moreover, the move starts at "time 0" by expressing an effective $s$-cycle $Z$ as a difference of effective $s$-cycles both of which have intersection properties no worse than $Z$. Finally, our Moving Lemma is applicable to smooth quasiprojective varieties, for it is stated for a possibly singular projective variety $X$ resulting in a conclusion of proper intersection off the singular locus of $X$.

The classical motivation for the moving lemma was to define an intersection product on algebraic cycles modulo rational equivalence, thereby establishing the Chow $\operatorname{ring} A_{*}(X)$. Apparently, the classical literature overlooked the question of whether or not intersection of cycles defined via a moving lemma is independent of the move (e.g., [Chev], [Chow], $[\mathrm{R}]$, [S2]). One direct consequence of our Moving Lemma is a proof for smooth quasiprojective varieties that the intersection product is indeed well defined independent of the choice of move (Theorem 3.4). Of course, the intersection product now has an intrinsic formulation for all smooth algebraic varieties due to Fulton and MacPherson [Fu]. We

*Partially supported by the N.S.F. and NSA Grant \# MDA904-93-H-3029

**Partially supported by the N.S.F. 
should emphasize that our arguments are projective in nature, and thus apply directly only to quasi-projective varieties.

We gratefully acknowledge helpful comments from William Fulton and Vladimir Voevodsky. We are especially indebted to Ofer Gabber who foresaw the validity of such a moving lemma for families and directed us to local estimates arising in the proof of the key Theorem 1.1.

\section{Definitions, Conventions, and a Brief Outline}

We adopt the following conventions thoughout. We fix a ground field $k$, choose an algebraic closure $\bar{k} \supset k$, and consider projective spaces $\mathbf{P}^{n}$ over $k$ of various dimensions $n$. A projective variety over $k$ is a reduced and absolutely irreducible algebraic $k$ scheme which admits a (Zariski) closed embedding in some projective space $\mathbf{P}^{n}$. A quasiprojective variety over $k$ is a Zariski open subset of some projective algebraic variety over $k$ whose complement has defining ideal also defined over $k$. The set of geometric points $X(\bar{k})$ of such a quasi- projective variety $X$ is the set of morphisms $\operatorname{Spec}(\bar{k}) \rightarrow X$ of schemes over $k$. For a point $x$ on a quasi-projective variety $X$, we denote by $\mathcal{O}_{X, x}$ the stalk at $x \in X$ of the structure sheaf of $X$ and by $\mathfrak{m}_{x}$ the maximal ideal of this local ring. An algebraic cycle $Z$ of dimension $r$ on a quasi-projective variety $X$ is an integral combination of closed, $r$-dimensional subvarieties of $X$, which are (reduced, irreducible but not necessarily absolutely irreducible and) defined over $k$. We say $Z$ is effective if the integer coefficients are all positive. Given a cycle $Z$ on $X$, we define its support to be the algebraic subset $|Z| \subset X$ consisting of the union of the irreducible components of $Z$. If $Y$ and $Z$ are cycles on $X$ of dimension $r$ and $s$ respectively with $r+s \geq m=\operatorname{dim}(X)$, we say that $Y$ and $Z$ intersect properly if each component of $|Y| \cap|Z|$ has dimension $\leq r+s-m$. If $X$ is smooth and if $Y$ and $Z$ intersect properly, then the intersection product $Y \bullet Z$ is a well- defined cycle of dimension $r+s-m$ on $X$ (See [Fu]).

Let $X \subset \mathbf{P}^{n}$ be a closed embedding over $k$ of an $m$ - dimensional projective variety $X$. For integers $d \geq 0$ and $r$ with $0 \leq r<\operatorname{dim}(X)$, we denote by $\mathcal{C}_{r, d}(X)$ the Chow variety of effective $r$-cycles of degree $d$ on $X$ (cf. [S1]). The disjoint union

$$
\mathcal{C}_{r}(X)=\coprod_{d \geq 0} \mathcal{C}_{r, d}(X)
$$

has the structure of an abelian monoid and is called the Chow monoid. The geometric points $\mathcal{C}_{r}(X)(\bar{k})$ of this Chow monoid constitute the (discrete) monoid of effective $r$-cycles on $X_{\bar{k}}$, the base-change of $X$ to $\operatorname{Spec}(\bar{k})$. (See $[\mathrm{F}, 1.2]$ for a discussion of the the $k^{\prime}$-rational points of $\mathcal{C}_{r}(X)$ for any $k^{\prime} / k$.)

Given an $r$-cycle $Y$ and an $s$-cycle $Z$ on a projective variety $X \subset \mathbf{P}^{n}$ of dimension $m$ with $r+s \geq m$, the classical Chow Moving Lemma (cf. [R]) asserts the existence of a rational equivalence between $Z$ and a necessarily ineffective $s$-cycle $Z^{\prime}$ which intersects $Y$ properly at all smooth points of $X$. Roughly speaking the proof proceeds as follows. One considers a linear projection $\pi_{L}: \mathbf{P}^{M} \longrightarrow \mathbf{P}^{m}$ whose vetex $L \cong \mathbf{P}^{M-m-1}$ does not meet $X$ and constructs the projecting cone $C_{L}(Z)=\pi_{L}^{*}\left(\pi_{L *}(Z)\right)$ of $Z$. Since $\left.\pi_{L}\right|_{X}$ is a finite morphism, $C_{L}(X) \bullet X$ is a well-defined $s$-cycle on $X$ which can be moved to intersect 
$Y$ properly by moving the cone $C_{L}(Z)$ in $\mathbf{P}^{M}$. The idea then is to choose $L$ so that the residual cycle

$$
R_{L}(Z)=C_{L}(Z) \bullet X-Z
$$

intersects $Y$ properly at least at all points outside the ramification locus of $\left.p_{L} \stackrel{\text { def }}{=} \pi_{L}\right|_{X}$. Furthermore, any component of excess intersection of $\left|R_{L}(Z)\right| \cap|Y|$ lies in $\operatorname{ram}\left(p_{L}\right) \cap|Z| \cap$ $|Y|$. Thus, if we choose $L_{1}, \ldots, L_{m+1}$ so that $\cap_{i} \operatorname{ram}\left(p_{L_{i}}\right)$ is contained in the singular locus of $X$, then after $m+1$ iterations the residual cycle $R_{L_{m+1}} \circ R_{L_{m}} \circ \cdots \circ R_{L_{1}}(Z)$ will meet $Y$ properly at all regular points of $X$.

In our paper we shall carry through this argument so that it applies simultaneously to all effective cycles $Y, Z$ of degree bounded by any specified positive integer $e$. To do this we consider the reimbeddings $X \hookrightarrow \mathbf{P}^{M_{d}}$ via the Veronese embeddings $\mathbf{P}^{M} \hookrightarrow \mathbf{P}^{M_{d}}$ of degree $d$. For each $d$ we consider the subset $\mathcal{U}_{X}(d)$ of linear subspaces of codimension $m+1$ in $\mathbf{P}^{M_{d}}$ which do not meet $X$. The main point is to show that the codimension of "bad $L$ 's" in $\mathcal{U}_{X}(d)$, i.e., those for which $R_{L}(Z)$ does not have an improved intersection with $Y$, goes to infinity as $d$ goes to infinity. This is achieved in $\S 1$.

In $\S 2$ we construct the moving of the projecting cones. This is conventionally done by projective transformations of the ambient $\mathbf{P}^{M}$. Here we introduce a different method which is related to the movings introduced in $[\mathrm{L}]$ and algebraicized in $[\mathrm{F}]$ to prove the "algebraic suspension theorem" in Lawson homology. This argument essentially verifies that effective $s$-cycles of bounded degree in $\mathbf{P}^{n}$ can be moved to intersect properly a hyperplane. Here we generalize this argument so that it applies to all effective $r$-cycles of bounded degree, where $s+r \geq n$, rather than to a single hyperplane.

In $\S 3$ we present our main results. In addition to Theorems 3.1 and 3.4 mentioned above, we prove in Theorem 3.5 that the intersection product on homotopy groups of cycle spaces on a smooth, projective complex variety (cf. [F-G]) can be represented by the intersection of families of cycles which have been moved to intersect properly. In Theorem 3.7, we present a basic ingredient of duality theorems for cycle spaces. It is the assertion that for a flat map $X \rightarrow B$ from a smooth variety, families of $s$-cycles on $X$ with $s \geq \operatorname{dim} B$ can be moved to be equidimensional over $B$.

\section{Residual cycles}

Consider an $m$-dimensional projective variety $X$ provided with a closed embedding $X \subset \mathbf{P}^{n}$ over $k$. Choose a positive integer $d$ and define

$$
\mathcal{U}_{X} \equiv \mathcal{U}_{X}(d) \subset \mathbf{P}\left(\Gamma\left(O_{\mathbf{P}^{n}}(d)^{m+1}\right)\right)
$$

to be the Zariski open set of those $\mathbf{F}=\left(f_{0}, \ldots, f_{m}\right)$ with the property that the zero locus $\mathcal{L}_{\mathbf{F}}=\left\{t \in \mathbf{P}^{n}: \mathbf{F}(t)=0\right\}$ misses $X$ and thus has codimension $m+1$ in $\mathbf{P}^{n}$. (More precisely, $\mathbf{F}$ is a $k$-rational point of the indicated Zariski open subset.) Each such F determines a finite morphism (defined over $k$ )

$$
p_{\mathbf{F}}: X \longrightarrow \mathbf{P}^{m}
$$


which can be viewed as follows. Let $v: \mathbf{P}^{n} \longrightarrow \mathbf{P}^{M}$ denote the Veronese embedding of degree $d$ (so $M=\left(\begin{array}{c}n+d \\ d\end{array}\right)-1$ ). Then each $\mathbf{F} \in \mathcal{U}_{X}$ determines a surjective linear projection

$$
\pi_{\mathbf{F}}: \mathbf{P}^{M}-->\mathbf{P}^{m}
$$

with the property that $p_{\mathbf{F}}=\left.\pi_{\mathbf{F}} \circ v\right|_{X}$. The condition $\mathbf{F}(x) \neq 0$ for $x \in X$ is equivalent to the condition that the linear subspace $L(\mathbf{F}) \stackrel{\text { def }}{=}$ the vertex of the projection $\pi_{\mathbf{F}}$, does not meet $v(X)$.

Suppose now that $Y, Z \subset X$ are closed algebraic subsets of pure dimension $r$ and $s$ respectively, where $r+s \geq m$. Let $Y \times Z-\Delta$ denote $(Y \times Z) \cap\left(X^{2}-\operatorname{diag}(X)\right)$ and denote by $Y \star_{\mathbf{F}} Z$ the following closed subset of $Y \times Z-\Delta$ :

$$
Y \star_{\mathbf{F}} Z \equiv\left\{(y, z): y \neq z, p_{\mathbf{F}}(y)=p_{\mathbf{F}}(z)\right\}
$$

for any $\mathbf{F} \in \mathcal{U}_{X}$. We begin by investigating the condition on $\mathbf{F} \in \mathcal{U}_{X}$ that $Y \star_{\mathbf{F}} Z$ should have pure dimension $r+s-m$.

An interesting special case of the following theorem is the case in which $Y$ equals $Z$, a cycle of dimension $r \geq m / 2$ on the projective variety $X$ of dimension $m$. Then, our theorem asserts for "most" projections of sufficiently high degree that the projection is injective on components of $Z$ off a subset of dimension no greater than $2 r-m$.

Theorem 1.1 Let $X \subset \mathbf{P}^{M}$ be a closed embedding over $k$ of an $m$-dimensional projective variety $X$. Consider closed algebraic subsets $Y, Z \subset X$ of pure dimension $r, s$ respectively, and assume that $r+s \geq m$. Then for any $k$-rational point $\mathbf{F} \in \mathcal{U}_{X}(d)$, each irreducible component of $Y \star_{\mathbf{F}} Z$ has dimension $\geq r+s-m$.

Fix any integer $N>0$, and suppose for each closed point $w=(y, z) \in Y \times Z-\Delta$ and for each $\varphi \in \Gamma\left(\mathcal{O}_{\mathbf{P}^{n}}(d)\right)$ with $\varphi(y) \neq 0 \neq \varphi(z)$ that the map

$$
\Gamma\left(\mathcal{O}_{\mathbf{P}^{n}}(d)\right) \longrightarrow \mathcal{O}_{Y, y} / \mathfrak{m}_{y}^{N+1} \times \mathcal{O}_{Z, z} / \mathfrak{m}_{z}^{N+1}
$$

sending $f$ to the restrictions of $f / \varphi$, is surjective. Then the subset

$$
\mathcal{B}(d)_{\{Y, Z\}} \subset \mathcal{U}_{X}(d)
$$

of those $\mathbf{F}$ for which some component of $Y \star_{\mathbf{F}} Z$ has dimension $>r+s-m$ is a (Zariski) closed subset of codimension at least $N-r-s$.

Proof. Fix $w=(y, z) \in Y \times Z-\Delta$ and denote by $\mathcal{B}_{w} \subset \mathcal{U}_{X} \equiv \mathcal{U}_{X}(d)$ the subset of those $\mathbf{F}=\left(f_{0}, \ldots, f_{m}\right)$ for which some component of $Y \star_{\mathbf{F}} Z$ has dimension $>r+s-m$ at $w$. Observe that the germ at $w$ of the variety $Y \star_{\mathbf{F}} Z$ is defined by the equations:

$$
f_{i}\left(y^{\prime}\right) f_{j}\left(z^{\prime}\right)=f_{j}\left(y^{\prime}\right) f_{i}\left(z^{\prime}\right) \quad \text { for } \quad 0 \leq i, j \leq m
$$

in a neighborhood of $w=(y, z)$ in $Y \times Z$. Now there exists a linear combination $\varphi=\sum a_{i} f_{i}$ of $f_{0}, \ldots, f_{m}$ with the property that $\varphi(y) \neq 0 \neq \varphi(z)$. For notational convenience we assume that $\varphi=f_{0}$, and we restrict attention to those $\mathbf{F} \in \mathcal{U}_{X}$ for which $f_{0}(y) \neq 0 \neq f_{0}(z)$. 
The general case will follow since $\mathcal{U}_{X}$ is covered by a finite collection of sets of this type. (In fact the set of linear combinations $f_{i}+f_{j}$ for $0 \leq i \leq j \leq m$ will do.)

With this assumption on $f_{0}$ one sees directly that equations (1.1.1) are equivalent to the equations:

$$
g_{\mathbf{F}}^{i}\left(y^{\prime}, z^{\prime}\right)=\frac{f_{i}\left(y^{\prime}\right)}{f_{0}\left(y^{\prime}\right)}-\frac{f_{i}\left(z^{\prime}\right)}{f_{0}\left(z^{\prime}\right)}=0 \quad \text { for } 1 \leq i \leq m
$$

on the germ of $Y \times Z$ at $w$. In particular, the germ at $w$ of $Y \star_{\mathbf{F}} Z$ has dimension $\geq r+s-m$.

We now proceed inductively to estimate the dimension of the bad set $\mathcal{B}_{w}$. We phrase the problem in terms of the local rings. In what follows, $\mathfrak{m}$ will always denote the maximal ideal of the local ring in question. Set $R(w, \mathbf{F})_{0}=\mathcal{O}_{Y \times Z, w}$, and let $R(w, \mathbf{F})_{i}$ denote the local ring $\mathcal{O}_{Y \times Z, w} /\left\langle g_{\mathbf{F}}^{1}, \ldots, g_{\mathbf{F}}^{i}\right\rangle$ for $1 \leq i \leq m$. If $R(w, \mathbf{F})_{i}$ has pure dimension $r+s-i$ for some $i<m$, then the condition on $g_{\mathbf{F}}^{i+1}$ that $R(w, \mathbf{F})_{i+1}$ have dimension $\leq r+s-i-1$ is the condition that $g_{\mathbf{F}}^{i+1}$ be non-zero in $R(w, \mathbf{F})_{i, \alpha} \equiv R(w, \mathbf{F})_{i} / \mathcal{P}_{i, \alpha}$ for each minimal prime $\mathcal{P}_{i, \alpha}$ of $R(w, \mathbf{F})_{i}$. This is guaranteed if the class of $g_{\mathbf{F}}^{i+1}$ is non-zero in the quotient $R(w, \mathbf{F})_{i, \alpha} / \mathfrak{m}^{N+1}$ for all $\alpha$.

Let us now fix $\varphi \in \Gamma\left(\mathcal{O}_{\mathbf{P}^{n}}(d)\right)$ with $\varphi(y) \neq 0 \neq \varphi(z)$ and set $\mathcal{B}_{w, \varphi}=\left\{\mathbf{F} \in \mathcal{B}_{w}: f_{0}=\right.$ $\varphi\}$. Consider the subset $\mathcal{B}_{w, \varphi}^{i+1} \subset \mathcal{B}_{w, \varphi}$ of those $\mathbf{F}$ for which $R(w, \mathbf{F})_{i}$ has pure dimension $r+s-i$ but $R(w, \mathbf{F})_{i+1}$ is not of dimension $r+s-(i+1)$. By the paragraph above we know that if $\mathbf{F} \in \mathcal{B}_{w, \varphi}^{i+1}$, then for some $\alpha, f_{i+1}$ lies in the kernel $K_{i+1, \alpha} \equiv \operatorname{ker}\left(G_{\alpha}^{i+1}\right)$ of the linear map

$$
G_{\alpha}^{i+1}: \Gamma\left(\mathcal{O}_{\mathbf{P}^{n}}(d)\right) \longrightarrow R(w, \mathbf{F})_{i, \alpha} / \mathfrak{m}^{N+1}
$$

defined by taking the image in $R(w, \mathbf{F})_{i, \alpha} / \mathfrak{m}^{N+1}$ of the germ at $w=(y, z)$ of the function

$$
G^{i+1}(\mathbf{F})\left(y^{\prime}, z^{\prime}\right)=\frac{f_{i+1}\left(y^{\prime}\right)}{\varphi\left(y^{\prime}\right)}-\frac{f_{i+1}\left(z^{\prime}\right)}{\varphi\left(z^{\prime}\right)}
$$

We make the following claim which is proved below.

\section{Claim:}

$$
\operatorname{codim}\left(K_{i+1, \alpha}\right) \geq N
$$

We now show how this claim proves the theorem. Consider the closed subvariety

$$
\mathcal{W} \equiv\left\{(y, z, \mathbf{F}):(y, z) \in\left(Y \star_{\mathbf{F}} Z\right)^{c l}\right\} \subset Y \times Z \times \mathcal{U}_{X}
$$

where $\left(Y \star_{\mathbf{F}} Z\right)^{c l} \subset Y \times Z$ denotes the closure of $Y \star_{\mathbf{F}} Z$. By upper semi-continuity of the dimension of the fibres of $\mathcal{W} \rightarrow \mathcal{U}_{X}$, we conclude that $\mathcal{B}(d)_{\{Y, Z\}} \subset \mathcal{U}_{X}$ is a closed subvariety. Moreover, if $\tilde{\mathcal{B}} \subset \mathcal{W}$ denotes $\operatorname{pr}_{3}^{-1}\left(\mathcal{B}(d)_{\{Y, Z\}}\right)$, then the fibre of $\tilde{\mathcal{B}}$ above $w \in(Y \times Z)-\Delta$ equals $\mathcal{B}_{w}$. Therefore if we prove that

$$
\text { The codimension of } \mathcal{B}_{w} \text { in }\{w\} \times \mathcal{U}_{X} \text { is } \geq N
$$

it will follow that the codimension of $\operatorname{pr}_{3}(\tilde{\mathcal{B}})=\mathcal{B}(d)_{\{Y, Z\}}$ in $\mathcal{U}_{X}$ is at least $N-r-s$ as claimed. 
To prove (1.1.6) we proceed as follows. Set $\Gamma=\Gamma\left(\mathcal{O}_{\mathbf{P}^{n}}(d)\right)$ and let $p: \mathcal{U}_{X} \subset$ $\Gamma^{\times(m+1)} \longrightarrow \Gamma$ denote projection onto the first coordinate. Then for $\varphi \in \Gamma$ with $\varphi(y) \neq$ $0 \neq \varphi(z)$ we see that

$$
\mathcal{B}_{w, \varphi}=p^{-1}(\varphi) \cap \mathcal{B}_{w} \cong \Gamma^{\times m} \cap \mathcal{B}_{w}
$$

It will suffice to prove that the codimension of $\mathcal{B}_{w, \varphi}$ in $\Gamma^{\times m}$ is $\geq N$. Note that

$$
\mathcal{B}_{w, \varphi}=\bigcup_{i=1}^{m} \mathcal{B}_{w, \varphi}^{i}
$$

and that $\mathcal{B}_{w, \varphi}^{1} \subset \bigcup_{\alpha} K_{1, \alpha}$. Now for $i>1$, the $K_{i, \alpha}$ depend on $\mathbf{F}^{k}$ for $k<i$. So fix $i>1$, let $p: \Gamma^{\times m} \rightarrow \Gamma^{\times i}$ be projection onto the first $i$ coordinates, and let $\mathcal{G}^{i}$ denote the complement of $p\left(\mathcal{B}_{w, \varphi}^{1} \cup \cdots \cup \mathcal{B}_{w, \varphi}^{i}\right) \subset \Gamma^{\times i}$. (Note that $\mathcal{G}^{i}$ consists of all $\mathbf{F}_{i}=\left(\mathbf{F}^{1}, \ldots, \mathbf{F}^{i}\right.$ ) such that $R(w, \mathbf{F})_{i}$ has pure dimension $\left.r+s-i\right)$. Then for each $\mathbf{F}_{i} \in \mathcal{G}^{i}$ we have that

$$
p^{-1}\left(\mathbf{F}_{i}\right) \cap \mathcal{B}_{w, \varphi}^{i+1} \subset\left\{\bigcup_{\alpha} K_{i+1, \alpha}\right\} \times \Gamma^{\times(m-i-1)} \subset \Gamma^{\times(m-i)}
$$

where the union over $\alpha$ is finite. Hence (1.1.6) follows from our claim (1.1.5).

Thus it remains to prove our claim (1.1.5) for $i=0, \ldots, m-1$. We proceed as follows. Denote by $R(y, \mathbf{F})_{i, \alpha}$ the quotient of the local ring $\mathcal{O}_{Y, y}$ by the pre-image of $\mathcal{P}_{i, \alpha}$ under the composition $\mathcal{O}_{Y, y} \longrightarrow \mathcal{O}_{Y \times Z, w} \longrightarrow R(w, \mathbf{F})_{i, \alpha}$. We define $R(z, \mathbf{F})_{i, \alpha}$ analogously. These rings correspond to the projections to $Y$ and $Z$ of the subvariety with generic point $\mathcal{P}_{i, \alpha}$. For $i<m$ one of these projections must have positive dimension (since the subvariety itself does). Hence, for $i<m$ at least one of $R(y, \mathbf{F})_{i, \alpha}, R(z, \mathbf{F})_{i, \alpha}$ has Krull dimension $\geq 1$. We assume wlog that this is true of $R(y, \mathbf{F})_{i, \alpha}$. Then (by further projection onto a generic line) we know that

$$
\operatorname{dim}\left\{R(y, \mathbf{F})_{i, \alpha} / \mathfrak{m}^{N+1}\right\} \geq N
$$

Let $\Gamma=\Gamma\left(\mathcal{O}_{\mathbf{P}^{n}}(d)\right)$ as above and note that the map $G^{i+1}: \Gamma \longrightarrow \mathcal{O}_{Y \times Z, w}$ defined in (1.1.4) factors through the subspace $\mathcal{O}_{Y, y} \times \mathcal{O}_{Z, z} \subset \mathcal{O}_{Y \times Z, w}$. Passing to quotients gives a linear map

$$
\hat{G}^{i+1}: \Gamma \longrightarrow \mathcal{O}_{Y, y} / \mathfrak{m}_{y}^{N+1} \times \mathcal{O}_{Z, z} / \mathfrak{m}_{z}^{N+1}\left(\subset \mathcal{O}_{Y \times Z, w} / \mathfrak{m}_{w}^{N+1}\right)
$$

which by our assumption is surjective. Write $\hat{G}^{i+1}=\left(\hat{G}_{1}^{i+1}, \hat{G}_{2}^{i+1}\right)$ with respect to the splitting above and let $\Gamma_{0}=\operatorname{ker}\left(\hat{G}_{2}^{i+1}\right)$. Then $\hat{G}_{1}^{i+1}: \Gamma_{0} \longrightarrow \mathcal{O}_{Y, y} / \mathfrak{m}_{y}^{N+1} \times\{0\}$ is surjective. This produces a commutative diagram

$$
\begin{array}{cccc}
\Gamma_{0} \longrightarrow \mathcal{O}_{Y, y} / \mathfrak{m}_{y}^{N+1} & \times \mathcal{O}_{Z, z} / \mathfrak{m}_{z}^{N+1} & \longrightarrow \mathcal{R}(w, \mathbf{F})_{i, \alpha} / \mathfrak{m}^{N+1} \\
\hat{G}_{1}^{i+1} \searrow & \uparrow & \uparrow \varphi
\end{array}
$$




$$
\mathcal{O}_{Y, y} / \mathfrak{m}_{y}^{N+1} \quad \stackrel{\pi}{\longrightarrow} \mathcal{R}(y, \mathbf{F})_{i, \alpha} / \mathfrak{m}^{N+1}
$$

where $\hat{G}_{1}^{i+1}$ and $\pi$ are surjective and where $\varphi$ is injective (since it corresponds to pull-back of functions from the image of projection of the subvariety). Our claim now follows from (1.1.7). This completes the proof.

In order to apply Theorem 1.1, we show in the following lemma that the surjectivity hypothesis of the proposition is satisfied for all sufficiently large degrees $d$.

Lemma 1.2. Retain the notation of Theorem 1.1. For each $N>0$, there exists some $d_{N}$ such that for all $d \geq d_{N}$ the map

$$
\Gamma\left(\mathcal{O}_{\mathbf{P}^{n}}(d)\right) \longrightarrow \mathcal{O}_{Y, y} / \mathfrak{m}_{y}^{N+1} \times \mathcal{O}_{Z, z} / \mathfrak{m}_{z}^{N+1}
$$

sending $f$ to the restrictions of $f / \varphi$ is surjective for all closed points $(y, z) \in Y \times Z-\Delta$, all $\varphi \in \Gamma\left(\mathcal{O}_{\mathbf{P}^{n}}(d)\right)$ with $\varphi(y) \neq 0 \neq \varphi(z)$.

Proof. Since $\mathcal{O}_{\mathbf{P}^{n}, y} \rightarrow \mathcal{O}_{Y, y}, \mathcal{O}_{\mathbf{P}^{n}, y} \rightarrow \mathcal{O}_{Z, z}$ are surjective local homomorphisms, it suffices to consider the special case $Y=\mathbf{P}^{n}=Z$. Moreover, base-changing by a field extension $\operatorname{Spec}\left(k^{\prime}\right) \rightarrow \operatorname{Spec}(k)$ merely tensors the map (1.2.1) with $k^{\prime}$ over $k$. Consequently, it suffices to assume that both $y, z$ are rational points of $\mathbf{P}^{n}$. By the two-point homogeneity of $\mathbf{P}^{n}$ under $P G L_{n+1}$, it thus suffices to verify the existence of $d_{N}$ for a single pair of (rational) points $(y, z) \in \mathbf{P}^{n} \times \mathbf{P}^{n}$.

The map sending $f$ to the restrictions of $f / \varphi$ in $\mathcal{O}_{Y, y} / \mathfrak{m}_{y}^{N+1}$ and $\mathcal{O}_{Z, z} / \mathfrak{m}_{z}^{N+1}$ has image whose dimension is independent of the choice of $\varphi$ with $\varphi(y) \neq 0 \neq \varphi(z)$ since the quotient of any two such choices of $\varphi$ is invertible in both $\mathcal{O}_{Y, y}$ and $\mathcal{O}_{Z, z}$. Hence, it will suffice to consider the case where $\varphi=X_{0}^{d}$ for some $X_{0} \in \Gamma\left(\mathcal{O}_{\mathbf{P}^{n}}(1)\right)$.

Let $R=k\left[X_{1} / X_{0}, \ldots, X_{n} / X_{0}\right]$ denote the coordinate algebra of the affine variety $\mathbf{A}^{n}=\mathbf{P}^{n}-\left\{X_{0}=0\right\}$, where we assume that $X_{0}(y) \neq 0 \neq X_{0}(z)$. Since $\mathfrak{m}_{y}^{N+1}, \mathfrak{m}_{z}^{N+1}$ are coprime in $R$, the Chinese Remainder Theorem implies that

$$
R \longrightarrow R / \mathfrak{m}_{y}^{N+1} \times R / \mathfrak{m}_{z}^{N+1}
$$

is surjective. Let $d_{N}$ be the minimal degree $d$ for which polynomials of degree $\leq d_{N}$ in $R$ map via this surjective map onto $R / \mathfrak{m}_{y}^{N+1} \times R / \mathfrak{m}_{z}^{N+1}$. Then for any $d \geq d_{N},(1.2 .1)$ is surjective.

Let $X$ be a projective variety provided with a closed embedding $X \subset \mathbf{P}^{n}$ over $k$ and consider non-negative integers $r, d$. For any non-negative integer e, set

$$
\mathcal{C}_{r, \leq e}(X)=\coprod_{d \leq e} C_{r, d}(X)
$$

In the next proposition we consider the Veronese embeddings of $X$ of degree $d$. We consider all cycles of fixed degree $\leq e$ (in the original embedding) and examine those 
projections which are "bad" for pairs of such cycles. The main assertion is that the codimension of these bad projections goes to infinity with $d$.

Proposition 1.3. Let $X \subset \mathbf{P}^{n}$ be a closed subvariety of dimension $m$, let $r, s$ be nonnegative integers with $r+s \geq m$, and let $e$ be a positive integer. There exist (Zariski) closed subsets $\mathcal{B}(d)_{e}$ of the quasi-projective variety $\mathcal{U}_{X}(d)$,

$$
\mathcal{B}(d)_{e} \subset \mathcal{U}_{X}(d) \subset \mathbf{P}\left(\Gamma\left(\mathcal{O}_{\mathbf{P}^{n}}(d)\right)\right)^{m+1},
$$

for $d>0$ with

$$
\lim _{d \rightarrow \infty} \operatorname{codim} \mathcal{B}(d)_{e}=\infty
$$

which satisfy the following property: for any effective $r$-cycle $Y$ on $X$ of degree $\leq e$ and any effective $s$-cycle $Z$ on $X$ also of degree $\leq e,|Y| \star_{\mathbf{F}}|Z|$ has pure dimension $r+s-m$ whenever $\mathbf{F}$ is a $k$-rational point of $\mathcal{U}_{X}(d)-\mathcal{B}(d)_{e}$.

Proof. For notational brevity, let $\mathcal{C}_{r, e}$ denote $\mathcal{C}_{r, \leq e}(X)$. We define

$$
\mathcal{W}_{e} \subset X^{2} \times \mathcal{C}_{r, e} \times \mathcal{C}_{s, e} \times \mathcal{U}_{X}(d)
$$

to be the closed subariety of those quintuples $(y, z, Y, Z, \mathbf{F})$ for which $y \times z$ lies in the closure of some component of $Y \star_{\mathbf{F}} Z$. The principle of upper semi-continuity applied to $\mathcal{W}_{e}$ over $\mathcal{C}_{r, e} \times \mathcal{C}_{s, e} \times \mathcal{U}_{X}(d)$ implies that

$$
\mathcal{V}_{e} \subset \mathcal{C}_{r, e} \times \mathcal{C}_{s, e} \times \mathcal{U}_{X}(d)
$$

is a closed subvariety, where $\mathcal{V}_{e}$ consists of those triples $(Y, Z, F)$ such that $Y \star_{\mathbf{F}} Z$ has some irreducible component of dimension $>m-r-s$. Thus,

$$
\mathcal{B}(d)_{e}=\operatorname{pr}_{3}\left(\mathcal{V}_{e}\right) \subset \mathcal{U}_{X}(d)
$$

is a closed subvariety of $\mathcal{U}_{X}(d)$.

Observe that the fibre of $\mathcal{V}_{e}$ above $(Y, Z) \in \mathcal{C}_{r} \times \mathcal{C}_{s}$ is $\mathcal{B}_{\{Y, Z\}}$ (in the notation of Proposition 1.1). Let $E_{r}=\operatorname{dim}\left\{\mathcal{C}_{r, e}\right\}, E_{s}=\operatorname{dim}\left\{\mathcal{C}_{s, e}\right\}$. By Theorem 1.1 and Lemma 1.2 , for any $N$ we may find $d_{N}$ so that $\mathcal{B}(d)_{\{Y, Z\}} \subset \mathcal{U}_{X}(d)$ has codimension at least $N-E_{r}-E_{s}-r-s$ for $d \geq d_{N}$. Hence, $\mathcal{B}_{e}(d)$ has codimension $\geq N-r-s$ for $d \geq d_{N}$.

Finally, if $Y, Z$ are effective $r, s$ cycles of degrees $\leq e$ and if $Y \star_{\mathbf{F}} Z$ has some component of dimension $>r+s-m$, then $(Y, Z, \mathbf{F})$ determines a point of $\mathcal{V}_{e}$ so that $\mathbf{F} \in \mathcal{B}(d)_{e}$.

Chow's classical technique of moving $Z$ to intersect $Y$ properly entails consideration of a Veronese embedding $\mathbf{P}^{n} \rightarrow \mathbf{P}^{M}$ and a linear plane $L \subset \mathbf{P}^{M}$ of codimension $m+1$ missing $X$. We let $\pi_{L}: \mathbf{P}^{M}--->\mathbf{P}^{m}$ denote the linear projection with center $L$ and $p_{L}: X \rightarrow \mathbf{P}^{m}$ denote the finite morphism given as the restriction of $\pi_{L}$ to $X$. Departing from the classical construction ( $\mathrm{cf}[\mathrm{R}]$ ), we define the projecting cone of a cycle $Z$ on $X$ to be the cycle

$$
C_{L}(Z) \stackrel{\text { def }}{=} \pi_{L}^{*}\left(p_{L *}(Z)\right) \subset \mathbf{P}^{M}
$$


where $p_{L *}$ denotes proper push-forward and $\pi_{L}^{*}$ denotes the algebraic join operation $(-) \# L$ sending a cycle $W$ on $\mathbf{P}^{m}$ to $W \# L$ on $\mathbf{P}^{m} \# L=\mathbf{P}^{M}$ (cf. [L,2.10]). This differs from the classical definition which is given by the discontinuous construction of sending an irreducible cycle $Z$ to $\pi_{L}^{*}\left(p_{L}(Z)\right)$, where $p_{L}(Z)$ is the irreducible, reduced image of $Z$.

Since $p_{L}$ is a finite map, the intersection product $C_{L}(Z) \bullet X$ is defined for all cycles $Z$ on $X$. We recall that $p_{L}: X \rightarrow \mathbf{P}^{m}$ is flat at any smooth point $x \in X$ (cf. [Mat,20.D]). Thus, if no component of $Z$ is contained in the singular locus of $X$, then $C_{L}(Z) \bullet X$ can alternatively be described as the closure in $X$ of $p_{L}^{n s *}\left(p_{L *}(Z)\right)$, where $p_{L}^{n s}: X_{n s} \rightarrow \mathbf{P}^{m}$ is the restriction to $p_{L}$ to the complement of the singular locus of $X$.

One considers the residual cycle

$$
R_{L}(Z) \stackrel{\text { def }}{=} C_{L}(Z) \bullet X-Z \text {. }
$$

This is a linear construction on cycles, sending effective cycles to effective cycles (cf. Lemma 1.6).

Given $Y$ and $Z$, one wants to choose $L$ so that $R_{L}(Z)$ has an improved intersection with $Y$. A key to this is the following lemma proved in $[\mathrm{R}]$ for the classical definition of residual cycle involving the classical projecting cone $\pi_{L}^{*}\left(p_{L}(Z)\right) \bullet X$. Denote by $\operatorname{ram}\left(p_{L}\right)$ the ramification locus of the finite map $p_{L}: X \longrightarrow \mathbf{P}^{m}$. (In particular $\operatorname{ram}\left(p_{L}\right)$ contains all the singular points of $X$.)

Proposition 1.4 (cf. [R; Lemma 6]) Let $Z, X$ and $L$ be as above and consider a geometric point $\underline{y}: \operatorname{Spec}(\bar{k}) \rightarrow\left|R_{L}(Z)\right|$ above a closed point $y \in\left|R_{L}(Z)\right|$. Then either:

(i) There exists a geometric point $\underline{z}: \operatorname{Spec}(\bar{k}) \rightarrow|Z|$ over a closed point $z \in|Z|$ with $\underline{z} \neq \underline{y}$ and $p_{L}(\underline{z})=p_{L}(\underline{y})$, or:

(ii) $y \in \operatorname{ram}\left(\bar{p}_{L}\right)$.

Proof. Since the construction of the residual cycle is additive, we may assume that $Z$ is irreducible. By definition of $R_{L}(Z)$, if $y \notin Z$, then there exists a point $z \in Z$ with $z \neq y$ and $p_{L}(z)=p_{L}(y)$. Thus, we may assume $y \in Z$.

If $\left[Z: p_{L}(Z)\right]>1$ (i.e., if the function field of $Z$ is a non-trivial extension of that of $\left.p_{L}(Z)\right)$, then either (i) is satisfied for some $\underline{z}: \operatorname{Spec}(\bar{k}) \rightarrow Z$ or in a formal neighborhood of $y$ at least two sheets of $Z$ (above a formal neighborhood of $p_{L}(y) \in \mathbf{P}^{m}$ ) intersect at $y$. This latter situation implies that in a formal neighborhood of $y \in X$ at least two sheets of $X$ intersect at $y$ so that $y \in \operatorname{ram}\left(p_{L}\right)$.

If $\left[Z: p_{L}(Z)\right]=1$ and if $Z$ is a component of $R_{L}(Z)$, then as shown in [R; Lemma 6 ] $p_{L}$ must ramify along all of $Z$. Namely, a "general" point of $Z$ is necessarily smooth on $Z$ and would also be smooth on $X$ if $p_{L}$ does not ramify along $Z$. At such a point, $p_{L}$ is etale and so the multiplicity of $Z$ in $p_{L}^{n s *}\left(p_{L *}(Z)\right)$ is 1 ; this contradicts the assumption that $Z$ is a component of $R_{L}(Z)$.

Finally, assume that $\left[Z: p_{L}(Z)\right]=1$ and that $Z$ is not a component of $R_{L}(Z)$. Since $y \in\left|R_{L}(Z)\right|, y$ lies in some component $R$ of $R_{L}(Z)$ not equal to $Z$. Then, in a formal neighborhood of $y \in\left|R_{L}(Z)\right|$ at least two sheets (one determined by $Z$ and another determined by $R$ ) intersect at $y$ so that $y \in \operatorname{ram}\left(p_{L}\right)$. 
As in (1.0.1) and (1.0.2), for each $(m+1)$-tuple $\mathbf{F}=\left(f_{0}, \ldots, f_{m}\right)$ of homogeneous forms of degree $d$ on $\mathbf{P}^{n}$, we consider the associated linear projection $\pi_{\mathbf{F}}: \mathbf{P}^{M}--->\mathbf{P}^{m}$ with center $L(\mathbf{F})$. If $X \subset \mathbf{P}^{n}$ is a subvariety of dimension $m$ and if $\mathbf{F} \in \mathcal{U}_{X}(d)$, we denote by $p_{\mathbf{F}}: X \rightarrow \mathbf{P}^{m}$ the finite map given by restricting $\pi_{\mathbf{F}}$ to $X \subset \mathbf{P}^{M}$ (embedded via the Veronese embedding). For any cycle $Z$ on $X$ we set

$$
C_{\mathbf{F}}(Z)=\pi_{\mathbf{F}}^{*}\left(p_{\mathbf{F} *}((Z))\right)=C_{L(\mathbf{F})}(Z) \quad \text { and } \quad R_{\mathbf{F}}(Z)=C_{\mathbf{F}}(Z) \bullet X-Z
$$

Corollary 1.5. Consider a projective variety $X$ of dimension $m$ provided with a closed embedding $X \subset \mathbf{P}^{n}$. Let $Y$ be an $r$-cycle and $Z$ an $s$-cycle on $X$ with $r+s-m \geq 0$. Assume $\mathbf{F} \in \mathcal{U}_{X}(d)$ satisfies the condition that $|Y| \star_{\mathbf{F}}|Z|$ has no component of excess dimension (i.e., $>r+s-m$ ). Then any component of $|Y| \cap\left|R_{\mathbf{F}}(Z)\right|$ of excess dimension must be contained in $|Y| \cap|Z| \cap \operatorname{ram}\left(p_{\mathbf{F}}\right)$.

Proof. By definition of $|Y| \star_{\mathbf{F}}|Z|$, any $y \in\left(|Y| \cap\left|R_{\mathbf{F}}(Z)\right|\right)-|Z|$ necessarily lies in $\operatorname{pr}_{|Y|}\left(|Y| \star_{\mathbf{F}}|Z|\right)$. By Proposition 1.5, any $y \in\left(|Y| \cap\left|R_{\mathbf{F}}(Z)\right|\right)-\operatorname{ram}\left(p_{\mathbf{F}}\right)$ admits a geometric point $(y, \underline{z}): \operatorname{Spec}(\bar{k}) \rightarrow|Y| \star_{\mathbf{F}}|Z|$ which projects to $y$ over $y$, so that $y$ also lies in $\operatorname{pr}_{|Y|}\left(|\bar{Y}| \star_{\mathbf{F}}|Z|\right)$. Thus, the complement of $|Y| \cap|Z| \cap \overline{\operatorname{ram}}\left(p_{\mathbf{F}}\right)$ in $|Y| \cap\left|R_{\mathbf{F}}(Z)\right|$ lies in $\operatorname{pr}_{|Y|}\left(|Y| \star_{\mathbf{F}}|Z|\right)$ whose components have dimensions less than or equal to that of the maximal dimension of the components of $|Y| \star_{\mathbf{F}}|Z|$.

We shall use the following property of the construction of residual cycles. We recall that a continuous algebraic map $f: X \rightarrow Y$ with $Y$ projective is a set-theoretic map on geometric points which is induced by a correspondence $\Gamma_{F} \subset X \times Y$ (cf. [F]).

Lemma 1.6. Let $\mathbf{F}$ be a $k$-rational point of $\mathcal{U}_{X}(d) \subset \mathbf{P}\left(\Gamma\left(\mathcal{O}_{\mathbf{P}^{n}}(d)\right)\right)^{m+1}$. Then $R_{\mathbf{F}}(Z)$ is an effective s-cycle on $X$ whenever $Z$ is itself an effective $s$-cycle on $X$. Moreover, sending $Z$ to $R_{\mathbf{F}}(Z)$ determines a continuous algebraic map

$$
R_{\mathbf{F}}(-): \mathcal{C}_{s, \leq e}(X) \rightarrow \mathcal{C}_{s, \leq e^{\prime}}(X)
$$

where $e^{\prime}$ is a positive integer depending upon $d, e$ and the degree of $X$.

Proof. The fact that $R_{\mathbf{F}}(-)$ sends effective cycles on $X$ to effective cycles on $X$ follows immediately from the observation that each of the operations in the definition of $R_{\mathbf{F}}(-)$ is a well defined operation on effective cycles.

We employ various functoriality properties proved in $[\mathrm{F}]$. The Veronese embedding $\nu: \mathbf{P}^{n} \rightarrow \mathbf{P}^{M}$ of degree $d$ determines a morphism

$$
\nu_{*}: \mathcal{C}_{s, \leq e}(X) \rightarrow \mathcal{C}_{s, \leq d^{s} e}(\nu(X))
$$

The linear projection $p_{\mathbf{F}}: \nu(X) \rightarrow \mathbf{P}^{m}$ determines a morphism

$$
p_{\mathbf{F} *}: \mathcal{C}_{s, \leq d^{s} e}(\nu(X)) \rightarrow \mathcal{C}_{s, \leq d^{s} e}\left(\mathbf{P}^{m}\right)
$$


The join construction determines a continuous algebraic map

$$
\pi_{\mathbf{F}}^{*}: \mathcal{C}_{s, \leq d^{s} e}\left(\mathbf{P}^{m}\right) \rightarrow \mathcal{C}_{s+M-m, \leq d^{s} e}\left(\mathbf{P}^{M}\right)
$$

Furthermore, intersection product in $\mathbf{P}^{M}$ determines a continuous algebraic map

$$
(-) \bullet X: \mathcal{C}_{s+M-m, \leq d^{s} e}\left(\mathbf{P}^{M}\right) \rightarrow \mathcal{C}_{s, \leq d^{s} e f}\left(\mathbf{P}^{M}\right)
$$

where $f$ equals the degree of $\nu(X)$. Finally, the additive structure of the Chow monoid is also given by continuous algebraic maps. Thus, sending $Z$ to $R_{\mathbf{F}}(Z) \equiv \pi_{\mathbf{F}}^{*}\left(p_{\mathbf{P}^{m_{*}}}(Z)\right) \bullet X-Z$ is a continuous algebraic map.

In the following theorem, we verify that an iteration of the residual cycle construction enables one to arrange (by choosing the degrees of projections sufficiently large) that the iterated residual cycle for $Z$ meets $Y$ properly for all effectives cycles $Y, Z$ of degree $\leq e$.

Theorem 1.7 Let $X \subset \mathbf{P}^{n}$ be a closed subvariety of dimension $m$, and let $U \subset X$ be the Zariski open subset consisting of the smooth points of $X$. For any $(m+1)$-tuple of positive integers $\mathbf{d}=\left(d_{0}, \ldots, d_{m}\right)$, there is a (Zariski) open dense subset

$$
\mathfrak{R}_{X}(\mathbf{d}) \subset \prod_{i=0}^{m} \mathcal{U}_{X}\left(d_{i}\right) \subset \prod_{i=0}^{m} \mathbf{P}\left(\Gamma\left(\mathcal{O}_{\mathbf{P}^{n}}\left(d_{i}\right)\right)^{m+1}\right)
$$

consisting of $(m+1)$-tuples $\mathbf{F}^{*} \equiv\left(\mathbf{F}^{0}, \ldots, \mathbf{F}^{m}\right)$ with the property that

$$
U \cap \operatorname{ram}\left(p_{\mathbf{F}^{0}}\right) \cap \ldots \cap \operatorname{ram}\left(p_{\mathbf{F}^{m}}\right)=\emptyset .
$$

For any non-negative integers $r, s$ with $r+s \geq m$ and any positive integer $e$, there exists a (Zariski) closed subset

$$
\mathfrak{B}(\mathbf{d})_{e} \subset \mathfrak{R}_{X}(\mathbf{d})
$$

such that

(a) The codimension of $\mathcal{B}(\mathbf{d})_{\mathbf{e}}$ can be made arbitrarily large for all appropriately chosen d which are sufficiently large (cf. (1.8) below).

(b) For all effective $r$-cycles $Y$ and $s$-cycles $Z$ of degree $\leq e$ on $X$ and for all $k$-rational points $\mathbf{F}^{*} \in \mathfrak{R}_{X}(\mathbf{d})-\mathfrak{B}(\mathbf{d})_{e}$, each component of

$$
|Y| \cap\left|R_{\mathbf{F}^{*}}(Z)\right| \quad, \quad R_{\mathbf{F}^{*}}(Z) \equiv R_{\mathbf{F}^{m}} \circ \cdots \circ R_{\mathbf{F}^{0}}(Z)
$$

of "excess" dimension (i.e., $>r+s-m$ ) is contained in the singular locus $X-U$.

Proof. We consider the (closed) incidence correspondence $\mathcal{J} \subset \mathcal{U}_{X}\left(d_{0}\right) \times X \ldots \times \mathcal{U}_{X}\left(d_{m}\right) \times$ $X$ of those $\left(\mathbf{F}^{0}, x_{0}, \ldots, \mathbf{F}^{m}, x_{m}\right)$ with $x_{j} \in \operatorname{ram}\left(p_{\mathbf{F}^{j}}\right)$ for all $j$. Then the complement of $\mathcal{R}_{X}(\mathbf{d})$ in $\mathcal{U}_{X}(\mathbf{d}) \equiv \prod_{j=0}^{m} \mathcal{U}_{X}\left(d_{j}\right)$ is given by

$$
\mathcal{U}_{X}(\mathbf{d})-\mathcal{R}_{X}(\mathbf{d})=\operatorname{pr}\{\mathcal{J}) \cap\left(\mathcal{U}_{X}(\mathbf{d}) \times \Delta(X)\right\},
$$


where pr is the proper projection pr : $\mathcal{U}_{X}\left(d_{0}\right) \times X \ldots \times \mathcal{U}_{X}\left(d_{m}\right) \times X \rightarrow \mathcal{U}_{X}(\mathbf{d})$ and $\Delta: X \rightarrow X^{m+1}$ is the diagonal embedding. Hence, $\mathcal{R}_{X}(\mathbf{d})$ is open.

For any smooth point of $x \in X$, those $\mathbf{F} \in \mathcal{U}_{X}(d)$ such that $p_{\mathbf{F}}$ ramifies at $x$ is a proper closed subset (defined by the condtion that $L_{\mathbf{F}}$ meet the tangent planes of $X$ at $x)$. Thus, the subset of those $\mathbf{F}$ for which $p_{\mathbf{F}}$ ramifies everywhere is a proper closed subset of $\mathcal{U}_{X}(d)$ (empty, if $k$ has characteristic 0 ). We readily see that $\mathcal{R}_{X}(\mathbf{d})$ is nonempty by observing that $\mathbf{F}^{*} \in \mathcal{R}_{X}(\mathbf{d})$ provided that $p_{\mathbf{F}^{0}}$ does not ramify everywhere and such that $p_{\mathbf{F}^{j}}$ does not ramify everywhere along any non-empty irreducible component of $\operatorname{ram}\left(p_{\mathbf{F}^{j-1}}\right) \cap \cdots \cap \operatorname{ram}\left(p_{\mathbf{F}^{0}}\right) \cap U$, for each $j$ with $0<j \leq m$.

The asserted $\mathfrak{B}(\mathbf{d})_{e}$ is the intersection with $\mathfrak{R}_{X}(\mathbf{d})$ of the Zariski closed subset

$$
\mathfrak{B}^{\prime}(\mathbf{d})_{e} \subset \mathcal{U}_{X}(\mathbf{d})
$$

constructed as the union over $j, 0 \leq j \leq m$, of closed subsets

$$
\mathcal{U}_{X}\left(d_{0}\right) \times \ldots \mathcal{U}_{X}\left(d_{j-1}\right) \times \mathcal{B}\left(d_{0}, \ldots, d_{j}\right)_{e} \times \ldots \mathcal{U}_{X}\left(d_{m}\right)
$$

for suitably defined closed subsets $\mathcal{B}\left(d_{0}, \ldots, d_{j}\right)_{e} \subset \mathcal{U}_{X}\left(d_{j}\right)$. Namely, $\mathcal{B}\left(d_{0}\right)_{e} \subset \mathcal{U}_{X}\left(d_{0}\right)$ is the closed subset given by Proposition 1.3 for $d_{0}, e$. By Lemma 1.6, the residual cycles $R_{\mathbf{F}}(Z)$ constructed for $\mathbf{F}_{0} \in \mathcal{U}_{X}\left(d_{0}\right)$ and effective cycles $Z$ on $X$ of degree bounded by $e$ are of degrees bounded by some $e_{1}$ (depending upon both $e$ and $d_{0}$ ). We define $\mathcal{B}\left(d_{0}, d_{1}\right)_{e} \subset \mathcal{U}_{X}\left(d_{1}\right)$ to be the closed subset given by Proposition 1.3 for $d_{1}, e_{1}$. Continuing inductively, we conclude by Lemma 1.6 that the residual cycles $R_{\mathbf{F}_{j-1}} \circ R_{\mathbf{F}_{0}}(Z)$ constructed for $\mathbf{F}_{0} \in \mathcal{U}_{X}\left(d_{0}\right), \ldots, \mathbf{F}_{j-1} \in \mathcal{U}_{X}\left(d_{j-1}\right)$ and effective cycles $Z$ on $X$ of degree bounded by $e$ are of degrees bounded by some $e_{j}$ (depending upon $e$ and $d_{0}, \ldots, d_{j-1}$ ). We define $\mathcal{B}\left(d_{0}, \ldots, d_{j}\right)_{e} \subset \mathcal{U}_{X}\left(d_{j}\right)$ to be the closed subset given by Proposition 1.3 for $d_{j}, e_{j}$.

By Corollary 1.5, any component of excess dimension of $|Y| \cap\left|R_{\mathrm{F}^{*}}(Z)\right|$ must be contained in $|Y| \cap|Z| \cap \operatorname{ram}\left(p_{\mathbf{F}^{m}}\right) \cap \ldots \cap \operatorname{ram}\left(p_{\mathbf{F}^{0}}\right)$ provided that $\mathbf{F}^{*} \notin \mathcal{B}^{\prime}(\mathbf{d})_{e}$. Thus, for $\mathbf{F}^{*} \in \mathfrak{R}_{X}(\mathbf{d}), \mathbf{F}^{*} \notin \mathcal{B}^{\prime}(\mathbf{d})_{e}$, any component of excess dimension of $|Y| \cap\left|R_{\mathbf{F}^{*}}(Z)\right|$ must be contained in $\operatorname{ram}\left(p_{\mathbf{F}^{m}}\right) \cap \ldots \cap \operatorname{ram}\left(p_{\mathbf{F}^{0}}\right) \subset X-U$.

By Proposition 1.3, for any integer $c$ we may choose each $d_{j}$ (depending as above on $e$ and the $d_{i}$ 's for $\left.i<j\right)$ so that $\mathcal{B}\left(d_{0}, \ldots, d_{j}\right)_{e} \subset \mathcal{U}_{X}\left(d_{j}\right)$ has codimension at least $c$. This insures that

$$
\mathfrak{B}(\mathbf{d})_{e} \equiv \mathfrak{B}^{\prime}(\mathbf{d})_{e} \cap \mathfrak{R}(\mathbf{d}) \subset \mathfrak{R}(\mathbf{d})
$$

also has codimension at least $c$.

Note 1.8. Note from the paragraph above that each $d_{j}$ may be chosen arbitrarily, so long as it is sufficiently large. This lower bound depends only on $c, e$ and the choice of the previous integers $d_{1}, \ldots, d_{j-1}$.

In $\S 3$ we will use Theorem 1.7 in conjunction with the following proposition which expresses a cycle $Z$ in terms of projecting cones and the cycle $R_{\mathrm{F}^{*}}(Z)$. This proposition follows immediately from Corollary 1.5 and Lemma 1.6. 
Proposition 1.9. Let $X \subset \mathbf{P}^{n}$ be a closed subvariety of dimension $m$, let $r, s$ be nonnegative integers with $r+s \geq m$, and let $e$ be a positive integer. Assume that $\mathbf{d}$ is chosen so that the codimension of $\mathcal{B}(\mathbf{d})_{e} \subset \mathcal{R}_{X}(\mathbf{d})$ is at least 1 and that the field $k$ is sufficiently large that there exists a $k$-rational point $\mathbf{F}^{*} \in \mathfrak{R}_{X}(\mathbf{d})-\mathfrak{B}(\mathbf{d})_{e}$. Choose some such $\mathbf{F}^{*}$. Consider the equality

$$
Z=(-1)^{m+1} R_{\mathbf{F}^{*}}(Z)+\sum_{i=1}^{m}(-1)^{i} \pi_{\mathbf{F}^{i}}^{*}\left\{p_{\mathbf{F}^{i} *}\left\{R_{\mathbf{F}^{i-1}} \circ \cdots \circ R_{\mathbf{F}^{0}}(Z)\right\}\right\} \bullet X
$$

and let $\psi_{\mathbf{F}^{*}}^{+}(Z), \psi_{\mathbf{F}^{*}}^{-}(Z)$ denote the positive and negative parts of the right hand side of (1.9.1). Then:

(a) For each effective s-cycle $Z$ on $X,\left(\psi_{\mathbf{F}^{*}}^{+}(Z), \psi_{\mathbf{F}^{*}}^{-}(Z)\right)$ is a pair of effective $s$-cycles on $X$ with the property that

$$
Z=\psi_{\mathbf{F}^{*}}^{+}(Z)-\psi_{\mathbf{F}^{*}}^{-}(Z) .
$$

(b) For all effective cycles $Y, Z$ on $X$ of dimension $r, s$ which are of degrees $\leq e$, any component of excess dimension of either $|Y| \cap\left|\psi_{\mathbf{F}^{*}}^{+}(Z)\right|$ or $|Y| \cap \psi_{\mathbf{F}^{*}}^{-}(Z) \mid$ lies in $|Y| \cap|Z|$.

(c) This construction determines a continuous algebraic map

$$
\psi_{\mathbf{F}^{*}}=\left(\psi_{\mathbf{F}^{*}}^{+}, \psi_{\mathbf{F}^{*}}^{-}\right): \mathcal{C}_{s}(X) \rightarrow \mathcal{C}_{s}(X)^{2} .
$$

Moreover, there is an integer $e^{\prime}$ such that

$$
\psi_{\mathbf{F}^{*}}\left(\mathcal{C}_{s, \leq e}(X)\right) \subset \mathcal{C}_{s, \leq e^{\prime}}(X)^{2} .
$$

\section{Moves in Projective Space}

In this section, we consider effective $r$-cycles $Y$ and effective $s$-cycles $Z$ on $\mathbf{P}^{m}$ of degree bounded by a fixed positive integer $e$, where $r+s \geq m$. We present a construction on all $s$-cycles on $\mathbf{P}^{m}$ which moves each such $Z$ so that the resulting cycle intersects properly each such $Y$. The method we employ generalizes a technique introduced in $[\mathrm{L}]$ to move $s$-cycles $Z$ of bounded degree to intersect properly a given hyperplane $L \equiv \mathbf{P}^{m-1} \subset \mathbf{P}^{m}$. One can interpret this method as taking the "algebraic suspension" $z_{1} \# Z \subset \mathbf{P}^{m+1}=z_{0} \# \mathbf{P}^{m}$ and observing that $z_{1} \# Z$ intersects $z_{0} \# L$ properly provided that $z_{1} \neq z_{0}$. One then observes that for most divisors $D \subset \mathbf{P}^{m+1}$ of sufficiently high degree the projection of $\left(z_{1} \# Z\right) \bullet D$ off $z_{0}$ meets $L$ properly.

Throughout this section, we consider non-negative integers $r, s \leq m$ with $r+s \geq m$. We set $t \equiv m-r$. We shall fix a linear embedding

$$
\mathbf{P}^{m} \subset \mathbf{P}^{m+t},(m+t=2 m-r)
$$


and some fixed linear subspace $L_{0} \subset \mathbf{P}^{m+t}$ of dimension $t-1$ missing $\mathbf{P}^{m}$. Thus,

$$
\mathbf{P}^{m+t}=L_{0} \# \mathbf{P}^{m}
$$

where -\#- denotes the "algebraic join".

We next introduce our "parameter space of moves" for $s$-cycles in $\mathbf{P}^{m}$.

Definition 2.1. For any $t$-tuple of positive integers $\mathbf{N}=\left(N_{1}, \ldots, N_{t}\right)$, let

$$
\mathcal{L}(\mathbf{N}) \subset \operatorname{Grass}_{t-1}\left(\mathbf{P}^{m+t}\right) \times \prod_{i=1}^{t} \mathbf{P}\left(\Gamma\left(\mathcal{O}_{\mathbf{P}^{m+t}}\left(N_{i}\right)\right)\right)
$$

denote the open set consisting of those $(L, \mathbf{D})$, where $\mathbf{D}=\left(D_{1}, \ldots, D_{t}\right)$ which satisfy

(a) $L \cap \mathbf{P}^{m}=\emptyset=L \cap L_{0}$,

(b) $\quad\left|D_{1}\right| \cap \ldots \cap\left|D_{t}\right| \cap L=\emptyset=\left|D_{1}\right| \cap \ldots \cap\left|D_{t}\right| \cap L_{0}$.

For any $L \in \operatorname{Grass}_{t-1}\left(\mathbf{P}^{m+t}\right)$, we denote by $\mathcal{L}_{L}(\mathbf{N})$ the Zariski dense open subset of $\prod_{i=1}^{t} \mathbf{P}\left(\Gamma\left(\mathcal{O}_{\mathbf{P}^{m+t}}\left(N_{i}\right)\right)\right)$ given as the fibre of the projection $\mathcal{L}(\mathbf{N}) \rightarrow \operatorname{Grass}_{t-1}\left(\mathbf{P}^{m+t}\right)$ above $L$.

Observe that for any $k$-rational point $(L, \mathbf{D}) \in \mathcal{L}(\mathbf{N})$, the intersection product $D_{1} \bullet$ $\cdots \bullet D_{t}$ is a well defined $n$-cycle on $\mathbf{P}^{m+t}$ with support $\left|D_{1}\right| \cap \cdots \cap\left|D_{t}\right|$ which meets $L \# Z$ properly and which misses $L_{0}$, the center of the projection $\pi_{0}$.

In the following proposition, we establish a constructible set of arbitrarily high codimension of "good moves" for effective $s$-cycles $Z$ of degree $\leq e$ which satisfy a single intersection condition depending upon a chosen $(t-1)$ - dimensional linear subspace $L$. We recall that a subset of a quasi-projective variety is said to be constructible if it can be written as a finite disjoint union of (Zariski) locally-closed subsets. The key property of constructible subsets is that any map $f: X \rightarrow Y$ of quasi-projective varieties sends a constructible subset $C \subset X$ to a constructible subset $f(C) \subset Y$.

Proposition 2.2. Let $L$ be a $(t-1)$-dimensional linear subspace of $\mathbf{P}^{m+t}$ satisfying $L \cap \mathbf{P}^{m}=\emptyset=L \cap L_{0}$. For a given positive integer $e$ and a $t$-tuple of positive integers $\mathbf{N}=\left(N_{1}, \ldots, N_{t}\right)$, there exists a constructible subset

$$
\mathcal{B}_{L}(\mathbf{N})_{e} \quad \subset \quad \mathcal{L}_{L}(\mathbf{N})
$$

such that

(a) For a constant $K$ depending only upon $X$ and $e$,

$$
\operatorname{codim} \mathcal{B}_{L}(\mathbf{N})_{e} \geq \min \left(N_{i}\right)-K
$$

(b) For every $k$-rational point $\mathbf{D}=\left(D_{1}, \ldots, D_{t}\right) \notin \mathcal{B}_{L}(\mathbf{N})_{e}$, the cycles

$$
\left|L_{0} \# Y\right| \cap|L \# Z| \cap\left|D_{1}\right| \cap \cdots \cap\left|D_{i-1}\right| \text { and }\left|D_{i}\right|
$$


meet properly for all $i \geq 0$ whenever $Y$ is an effective $r$-cycle and $Z$ is an effective $s$-cycle both of degree $\leq e$ on $\mathbf{P}^{m}$ with the property that $L_{0} \# Y$ intersects $L \# Z$ properly.

Proof. Let

$$
\mathcal{J} \subset \mathcal{C}_{r, \leq e}\left(\mathbf{P}^{m}\right) \times \mathcal{C}_{s, \leq e}\left(\mathbf{P}^{m}\right) \times \mathbf{P}^{m+t}
$$

denote the incidence correspondence of triples $(Y, Z, x)$ satisfying $x \in\left|L_{0} \# Y\right| \cap|L \# Z|$. We define

$$
\mathcal{G}_{L, e} \quad \subset \quad \mathcal{C}_{r, \leq e}\left(\mathbf{P}^{m}\right) \times \mathcal{C}_{s, \leq e}\left(\mathbf{P}^{m}\right)
$$

to be the open subset above which the fibre of the projection

$$
\mathcal{J} \rightarrow \mathcal{C}_{r, \leq e}\left(\mathbf{P}^{m}\right) \times \mathcal{C}_{s, \leq e}\left(\mathbf{P}^{m}\right)
$$

has proper dimension (i.e., $r+s-m+t=s$ ). Note that $\mathcal{G}_{L, e}$ consists of pairs of cycles $(Y, Z)$ such that $L_{0} \# Y$ and $L \# Z$ intersect properly. We consider the closed subset

$$
\tilde{\mathcal{B}}_{L}(\mathbf{N})_{e} \subset \mathcal{G}_{L, e} \times \mathcal{L}_{L}(\mathbf{N})
$$

consisting of those tuples $(Y, Z, \mathbf{D})$ satisfying the condition that $L_{0} \# Y$ and $L \# Z$ intersect properly but

$$
\left|L_{0} \# Y\right| \cap|L \# Z| \cap\left|D_{1}\right| \cap \cdots \cap\left|D_{i-1}\right|, \quad\left|D_{i}\right|
$$

intersect improperly for some $i \leq t$. Finally, we set

$$
\mathcal{B}_{L}(\mathbf{N})_{e}=\operatorname{pr}_{3 *}\left[\left(\tilde{\mathcal{B}}_{L}\right)(\mathbf{N})_{e}\right] .
$$

We proceed to verify that the fibre $\tilde{\mathcal{B}}_{Y, Z} \subset \mathcal{L}_{L}(\mathbf{N})$ of $\tilde{\mathcal{B}}_{L}(\mathbf{N})_{e}$ above any $\left(Y, Z \in \mathcal{G}_{L, e}\right.$ has codimension at least $N$ if each $N_{i} \geq N$. This will immediately imply the codimension of $\mathcal{B}_{L}(\mathbf{N})_{e} \subset \mathcal{L}_{L}(\mathbf{N})$ is greater than some prescribed positive integer $c$ provided that

$$
N \geq c+\operatorname{dim}\left(\mathcal{C}_{r, \leq e}\left(\mathbf{P}^{m}\right)\right)+\operatorname{dim}\left(\mathcal{C}_{s, \leq e}\left(\mathbf{P}^{m}\right)\right) .
$$

The condition that $W_{j} \equiv\left|L_{0} \# Y\right| \cap|L \# Z| \cap\left|D_{1}\right| \cap \ldots \cap\left|D_{j-1}\right|$ meet $\left|D_{j}\right|$ improperly is equivalent to the condition that the restriction map

$$
\mathcal{O}_{P^{m+t}}\left(N_{j}\right) \longrightarrow \mathcal{O}_{W_{j}}\left(N_{j}\right) \longrightarrow \mathcal{O}_{W_{j, \omega}}\left(N_{j}\right)
$$

sends $D_{j} \in \Gamma\left(\mathcal{O}_{P^{m+t}}\left(N_{j}\right)\right)$ to 0 for some irreducible component $W_{j, \omega} \subset W_{j}$. Since $W_{j, \omega}$ has positive dimension, this composition has rank at least $N$. To see this note that if we restrict to some affine open $\mathbf{A}^{m} \subset \mathbf{P}^{m}$ containing a dense open subset of $W_{j, \omega}$, then the dimension of the subspace of regular functions on $\mathbf{A}^{m} \cap W_{j, \omega}$ of degree $\leq N$ is at least $N$. We conclude that the closed subvariety $\tilde{\mathcal{B}}_{Y, Z, j} \subset \mathcal{L}_{L}(\mathbf{N})$ consisting of those $\mathbf{D}$ with $D_{j}$ meeting $W_{j}$ improperly and $D_{j^{\prime}}$ meeting $W_{j^{\prime}}$ properly for $j^{\prime}<j$ has codimension at least $N$. Thus,

$$
\tilde{\mathcal{B}}_{Y, Z}=\coprod_{j} \tilde{\mathcal{B}}_{Y, Z, j} \subset \mathcal{L}_{L}(\mathbf{N})
$$


also has codimension at least $N$.

Proposition 2.2 suggests the following construction of moving cycles in $\mathbf{P}^{m}$, sending the $s$-cycle $Z$ to $\varphi_{L, \mathbf{D}}(Z)$.

Proposition 2.3 Let $\pi_{0}: \mathbf{P}^{m+t}--->\mathbf{P}^{m}$ denote the linear projection with center $L_{0}$. For any $e>0$ and any $t$-tuple $\mathbf{N}=\left(N_{1}, \ldots, N_{t}\right)$, the assignment

$$
\varphi_{L, \mathbf{D}}(Z) \stackrel{\text { def }}{=} \pi_{0 *}\left((L \# Z) \bullet D_{1} \bullet \ldots \bullet D_{t}\right)
$$

determines a continuous algebraic map

$$
\Phi: \mathcal{C}_{s}\left(\mathbf{P}^{m}\right) \times \mathcal{L}(\mathbf{N}) \longrightarrow \mathcal{C}_{s}\left(\mathbf{P}^{m}\right)
$$

with $\Phi\left(\mathcal{C}_{s, \leq e}\left(\mathbf{P}^{m}\right) \times \mathcal{L}(\mathbf{N})\right) \subset \mathcal{C}_{s, \leq e|\mathbf{N}|}\left(\mathbf{P}^{m}\right)$, where $|\mathbf{N}| \stackrel{\text { def }}{=} \prod_{i} N_{i}$. This construction has the following properties:

(a) For any $k$-rational point $(L, \mathbf{D}) \in \mathcal{L}(\mathbf{N})$ and any effective $s$-cycle $Z$ on $\mathbf{P}^{m}$, $\varphi_{L, \mathbf{D}}(Z)$ is also an effective $s$-cycle on $\mathbf{P}^{m}$.

(b) For any $k$-rational point $(L, \mathbf{D}) \in \mathcal{L}(\mathbf{N})$ with the property that $D_{1} \bullet \ldots \bullet D_{t}=$ $|\mathbf{N}| \cdot \mathbf{P}^{m}$

$$
\varphi_{L, \mathbf{D}}(Z)=|\mathbf{N}| \cdot Z
$$

for all effective $s$-cycles $Z$.

(c) If $(L, \mathbf{D})$ is a $k$-rational point of $\mathcal{L}(\mathbf{N})$ such that $\mathbf{D} \notin \mathcal{B}_{L}(\mathbf{N})_{e}(c f .(2.2))$, if $Y, Z$ are effective cyles on $\mathbf{P}^{m}$ of dimension $r, s$ and degree $\leq e$, and if $L_{0} \# Y$ meets $L \# Z$ properly, then

$$
Y, \varphi_{L, \mathbf{D}}(Z)
$$

intersect properly.

Proof. If $Z$ on $\mathbf{P}^{m}$ is an $s$-effective cycle of degree $d$, then $L \# Z$ is an effective $(s+t)$-cycle $\mathbf{P}^{m+t}$ also of degree $d$. Consequently, $(L \# Z) \bullet D_{1} \bullet \ldots \bullet D_{t}$ and thus also $\varphi_{L, \mathbf{D}}(Z)$ have degree $d \cdot|\mathbf{N}|$. Moreover, sending $\langle Z,(L, \mathbf{D})\rangle$ to $\varphi_{L, \mathbf{D}}(Z)$ determines a continuous algebraic map, for $\Phi$ is a composition of operations each of which is given by a continuous algebraic $\operatorname{map}$ (cf. $[\mathrm{F}]$ ).

As observed following Definition 2.1, the intersection product $D_{1} \bullet \cdots \bullet D_{t}$ is a well defined $n$-cycle on $\mathbf{P}^{m+t}$ with support $\left|D_{1}\right| \cap \cdots \cap\left|D_{t}\right|$ which meets $L \# Z$ properly and which misses $L_{0}$ for any $k$-rational point $(L, \mathbf{D}) \in \mathcal{L}(\mathbf{N})$. Thus, $\varphi_{L, \mathbf{D}}(Z)$ is a well defined $s$-cycle on $\mathbf{P}^{m}$.

To prove that $\varphi_{L, \mathbf{D}}(Z)=|\mathbf{N}| \cdot Z$ whenever $D_{1} \bullet \ldots \bullet D_{t}=|\mathbf{N}| \cdot \mathbf{P}^{m}$, observe that $\mathbf{P}^{m} \subset \mathbf{P}^{m+t}$ meets $L \# Z$ properly (since $L \cap \mathbf{P}^{m}=\emptyset$ ) and transversely at all regular points of $Z$.

If $(L, \mathbf{D}) \in \mathcal{L}(\mathbf{N})$, then $L_{0} \# Y, L \# Z$ have pure dimension $m, s+t$ respectively. The hypothesis that $L_{0} \# Y$ meets $L \# Z$ properly thus implies that $\left|L_{0} \# Y\right| \cap|L \# Z|$ has dimension $s$. We thus may apply Proposition 2.2 to conclude that if $\mathbf{D} \notin \mathcal{B}(\mathbf{N})_{e}$, if $Y, Z$ are effective 
cycles of degrees $\leq e$, and if $L_{0} \# Y$ meets $L \# Z$ properly, then $\left(L_{0} \# Y\right) \bullet(L \# Z) \bullet D_{1} \bullet \ldots \bullet D_{t}$ has pure dimension $r+s-m$. Because $L_{0} \cap\left|D_{1}\right| \cap \ldots \cap\left|D_{t}\right|=\emptyset, \pi_{0 *}$ restricts to a (well defined) finite map on $\left|(L \# Z) \bullet D_{1} \bullet \ldots \bullet D_{t}\right|$ We apply the projection formula to $\pi_{0 *}$ to conclude that

$$
Y \bullet \pi_{0 *}\left[(L \# Z) \bullet D_{1} \bullet \ldots \bullet D_{t}\right]=\pi_{0 *}\left[\left(L_{0} \# Y\right) \bullet(L \# Z) \bullet D_{1} \bullet \ldots \bullet D_{t}\right]
$$

Hence, $Y, \varphi_{L, \mathbf{D}}(Z)$ meet properly.

The following lemma will enable us to apply Proposition 2.3 to all effective cycles of bounded degree.

Lemma 2.4. Let $L$ be a $k$-rational point of $\operatorname{Grass}_{t-1}\left(\mathbf{P}^{m+t}\right)$ satisfying $L \cap P^{m}=\emptyset=$ $L \cap L_{0}$. Then

$$
\left(L_{0} \# Y\right),(L \# Z) \text { intersect properly }
$$

in $\mathbf{P}^{m+t}$ for every $r$-cycle $Y$, s-cycle $Z$ on $\mathbf{P}^{m}$ such that either $\left|L_{0} \# Y\right| \cap L=\emptyset$ or $Y$ intersects $Z$ properly.

Proof. We first check that if $Y, Z$ intersect properly, so do $\left(L_{0} \# Y\right),(L \# Z)$ for any choice of $L$. Observe that

$$
\left|L_{0} \# Y\right| \cap P^{m}=|Y|, \quad|L \# Z| \cap P^{m}=|Z| .
$$

Thus, if some component of $\left|L_{0} \# Y\right| \cap|L \# Z|$ has dimension $>s$, then some component of $\left|L_{0} \# Y\right| \cap|L \# Z| \cap P^{m}=|Y| \cap|Z|$ would have dimension $>s-t$.

On the other hand, if $\left|L_{0} \# Y\right| \cap L=\emptyset$, then $L \# z$ intersects $L_{0} \# Y$ in only finitely many points for any $z \in \mathbf{P}^{m}$. Thus, $\left|L_{0} \# Y\right| \cap|L \# Z|$ has dimension at most $s$.

Combining Proposition 2.3 and Lemma 2.4, we now verify for any given $e$ that we can "move" all effective $s$-cycles $Z$ of degree $\leq e$ so that the resulting cycles intersect properly every effective $r$-cycle $Y$ of degree $\leq e$.

As in Proposition 1.9, we require the existence of $k$-rational points avoiding certain "bad" closed subsets of positive codimension. The existence of such $k$-rational points is automatic for an infinite field, but for a finite field all rational points of a given variety might lie on a subvariety of positive codimension. On the other hand, if $k$ is a finite field and if there are only finitely many "bad" subvarieties of positive codimension, there will exist some finite extension $k^{\prime}$ of $k$ such that there exist $k^{\prime}$-rational points avoiding these "bad sets". Moreover, any extension $k^{\prime \prime}$ of $k^{\prime}$ will evidently have this same property.

Since our definitions of "good" and "bad" sets are independent of $k$, we conclude that all "sufficiently large" finite fields $k$ of a given residue characteristic admit the existence of $k$-rational points missing the "bad" sets. We shall use the expression " $k$ is a sufficiently large finite field" to mean that there exist $k$-rational points avoiding the bad sets (of positive codimension) occuring in our constructions.

Proposition 2.5. Fix positive integers $e$ and $c$. Assume that either $k$ is an infinite field or a sufficiently large finite field. Consider a sequence $\mathbf{N}_{1}, \mathbf{N}_{2}, \mathbf{N}_{3}, \ldots$ of $t$-tuples of positive 
integers, and set $e_{1}=e$ and $e_{j+1}=\left|\mathbf{N}_{j}\right| e_{j}$ for $j>1$. Assume that the $\mathbf{N}_{j}$ 's are chosen so that $\min _{1 \leq i \leq t} N_{j+1, i}>K\left(e_{j}\right)+c$ where $K\left(e_{j}\right)$ is the constant in Proposition 2.2a. Then there exist an integer $E \leq \operatorname{dim}_{s, \leq e}\left(\mathbf{P}^{m}\right)$ and $(t-1)$-dimensional linear subspaces $L_{1}, \ldots, L_{E}$ of $\mathbf{P}^{m+t}$, each missing $L_{0}$ and $\mathbf{P}^{m}$, with the following properties:

(a) For each $j$,

$$
\operatorname{codim}\left\{\mathcal{L}_{L_{j}}\left(\mathbf{N}_{j}\right)-\mathcal{B}_{L_{j}}\left(\mathbf{N}_{j}\right)_{e_{j}}\right\} \geq c
$$

(b) For any $k$-rational point

$$
\left(\mathbf{D}_{1}, \ldots, \mathbf{D}_{E}\right) \in \prod_{j=1}^{E} \mathcal{L}_{L_{j}}\left(\mathbf{N}_{j}\right)-\mathcal{B}_{L_{j}}\left(\mathbf{N}_{j}\right)_{e_{j}}
$$

and any effective cycles $Y, Z$ on $\mathbf{P}^{m}$ of dimensions $r, s$ and degrees $\leq e$,

$$
Y \quad, \quad \varphi_{L_{E}, \mathbf{D}_{E}} \circ \cdots \circ \varphi_{L_{1}, \mathbf{D}_{1}}(Z)
$$

intersect properly.

Proof. Note to begin that assertion (a) follows immediately from Proposition 2.2a, so it remains to prove assertion (b).

For each (of the finitely many) irreducible components $A_{\gamma}$ of $A=C_{r, \leq e}\left(\mathbf{P}^{m}\right)$ of maximal dimension $d_{e}$, choose some $k$-rational point $a(\gamma) \in A_{\gamma}$ (possible since $k$ is assumed either infinite or sufficiently large). Using once again the fact that $k$ is infinite or sufficiently large, we may choose some $(t-1)$-dimensional linear plane $L_{1}$ in $\mathbf{P}^{m+t}$ missing $P^{m}, L_{0}$, and each $L_{0} \# Y_{a(\gamma)}$. Let $A_{1} \subset A$ denote the closed subvariety of those $Y$ 's such that $L_{1} \cap\left|L_{0} \# Y\right| \neq \emptyset$, and observe that each component of $A_{1}$ has dimension strictly less than $d_{e}$. By Proposition 2.3 and Lemma 2.4, $Y$ and $\varphi_{L_{1}, \mathbf{D}_{1}}(Z)$ intersect properly for any effective $r$-cycle $Y$ of degree $\leq e$ and any effective $s$-cycle $Z$ of degree $\leq e$ which does not lie in $A_{1}$ whenever $\mathbf{D}_{1}$ is $k$-rational point of $\mathcal{L}_{L_{1}}\left(\mathbf{N}_{1}\right)-\mathcal{B}_{L_{1}}\left(\mathbf{N}_{1}\right)_{e_{1}}$.

Proceeding inductively, assume for some $j \geq 1$ that we have defined the closed subvariety $A_{j} \subset A$ of codimension at least $j$ which satisfies the condition that

$$
Y, \varphi_{L_{j}, \mathbf{D}_{j}} \circ \cdots \circ \varphi_{L_{1}, \mathbf{D}_{1}}(Z)
$$

intersect properly for any effective $r$-cycle $Y$ of degree $\leq e$ and any effective $s$-cycle $Z$ of degree $\leq e$ which does not lie in $A_{j}$. For each (of the finitely many) irreducible components $A_{j, \gamma}$ of maximal dimension choose as above some point $a(\gamma) \in A_{j, \gamma}$ and choose $L_{j+1}$ missing $P^{m}, L_{0}$, and each $L_{0} \# Y_{a(\gamma)}$. We set $A_{j+1} \subset A_{j}$ equal to the closed subvariety of that $Y$ 's such that $L_{j+1} \cap\left|L_{0} \# Y\right|=\emptyset$. Applying Proposition 2.3 and Lemma 2.4 once again, we conclude that

$$
Y, \varphi_{L_{j}, \mathbf{D}_{j}} \circ \cdots \circ \varphi_{L_{1}, \mathbf{D}_{1}}(Z)
$$

intersect properly for any effective $r$-cycle $Y$ of degree $\leq e$ and any effective $s$-cycle $Z$ of degree $\leq e$ which does not lie in $A_{j}$, whenever $\mathbf{D}_{j}$ is $k$-rational point of $\mathcal{L}_{L_{j}}\left(\mathbf{N}_{j}\right)-$ $\mathcal{B}_{L_{j}}\left(\mathbf{N}_{j}\right)_{e_{j}}$. 
Finally, we see that this process stops after $E$ steps, where $E \leq d_{e}+1$.

The following theorem is our "Moving Lemma for Cycles of Bounded Degree" in the special case in which the variety $X$ is projective space $\mathbf{P}^{m}$ itself.

Theorem 2.6. Fix positive integers $e$ and $c$. Assume that either $k$ is an infinite field or a sufficiently large finite field. Choose $\mathbf{N}_{1}, \ldots, \mathbf{N}_{E}$ and $L_{1}, \ldots, L_{E}$ as in Proposition 2.5 and let

$$
\underline{0} \stackrel{\text { def }}{=}\left(\mathbf{D}_{1}, \ldots, \mathbf{D}_{E}\right) \in \prod_{j=1}^{E} \mathcal{L}_{L_{j}}\left(\mathbf{N}_{j}\right)
$$

be a $k$-rational point such that $D_{j, 1} \bullet \cdots \bullet D_{j, t}=\left|\mathbf{N}_{j}\right| \cdot \mathbf{P}^{m}$ for each $j, 1 \leq j \leq E$. (For example, suppose that $D_{j, i}=N_{j, i} H_{j, i}$ where $H_{j, 1}, \ldots, H_{j, t}$ are $k$-rational hyperplanes in general position.) Let

$$
\ell \subset \prod_{j=1}^{E} \prod_{i=1}^{t} \mathbf{P}\left(\Gamma\left(\mathcal{O}_{\mathbf{P}^{m+t}}\left(N_{j, i}\right)\right)\right)
$$

be any $k$-rational line through $\underline{0}$ chosen so that

$$
\ell^{\circ} \equiv \ell \cap \prod_{j=1}^{E} \mathcal{L}_{L_{j}}\left(\mathbf{N}_{j}\right)-\mathcal{B}_{L_{j}}\left(\mathbf{N}_{j}\right)_{e_{j}} \subset \ell
$$

is dense. Then there exists a continuous algebraic map

$$
\Theta: \mathcal{C}_{s}\left(\mathbf{P}^{m}\right) \times \ell^{\circ} \rightarrow \mathcal{C}_{s}\left(\mathbf{P}^{m}\right)
$$

with the following properties. Set $\theta_{p}=\left.\Theta\right|_{\mathcal{C}_{s}\left(\mathbf{P}^{m}\right) \times\{p\}}$.

(a) $\theta_{\underline{0}}(Z)=\prod_{j=1}^{E}\left|\mathbf{N}_{j}\right| \cdot Z$ for any effective s-cycle $Z$.

(b) For any $k$-rational point $p \in \ell^{\circ}, \theta_{p}$ determines a linear map on effective $s$-cycles on $\mathbf{P}^{m}$.

(c) For any effective s-cycle $Z$, the restriction of $\Theta$ to $\{Z\} \times \ell^{\circ}$ determines a rational equivalence $Z_{\theta} \subset \mathbf{P}^{m} \times \ell$ whose fibre above a point $p \in \ell^{\circ}$ is the cycle with Chow point $\theta_{p}(Z)$.

(d) For all effective cycles $Y, Z$ on $\mathbf{P}^{m}$ of dimensions $r, s$ and degrees $\leq e$ and all $k$-rational points $p \in \ell^{\circ}-\underline{0}$,

$$
Y, \theta_{p}(Z)
$$

intersect properly.

Proof. Iterating the continuous algebraic map $\Phi$ of Proposition 2.3, we obtain the continuous algebraic map

$$
\tilde{\Theta}: \mathcal{C}_{s}\left(\mathbf{P}^{m}\right) \times \mathcal{L}\left(\mathbf{N}_{1}\right) \times \cdots \times \mathcal{L}\left(\mathbf{N}_{1}\right) \rightarrow \mathcal{C}_{s}\left(\mathbf{P}^{m}\right)
$$


We define $\Theta$ to be the restriction of $\tilde{\Theta}$ to $\mathcal{C}_{s}\left(\mathbf{P}^{m}\right) \times \ell^{\circ}$.

Property (a) is given by Proposition 2.3.b, whereas property (b) follows from Proposition 2.3.a. To verify property (c), we let $\lambda$ denote the generic point of $\ell$ and $k(\lambda)$ denote the function field of $\ell$. Then $\Theta(Z, \lambda)$ determines a cycle on $\mathbf{P}^{m}$ rational over some radiciel extension of $k(\lambda)$ (cf. $[\mathrm{F}]$ ). On the other hand, since the operations used to define $\Theta$ send cycles to cycles, we see that the cycle $Z_{\lambda}$ with Chow point $\Theta(Z, \lambda)$ is in fact a cycle on $\mathbf{P}_{k(\lambda)}^{m}$. The closure of this cycle, $Z_{\theta}$ on $\mathbf{P}^{m} \times \ell$, is the asserted rational equivalence. (Observe that $Z_{\theta}$ is necessarily flat over $\ell$ so that the fibre $p \in \ell$ is given by specialization of $Z_{\lambda}$.) Finally, property (d) follows from Proposition 2.5.

\section{Main Theorem and Consequences}

Using Theorem 1.7, Proposition 1.9, and Theorem 2.6, we now prove our "Moving Lemma for Cycles of Bounded Degree".

Theorem 3.1. Let $X \subset \mathbf{P}^{n}$ be a projective variety of dimension $m$. Assume that either $k$ is an infinite field or a sufficiently large finite field. Let $r, s, e$ be non-negative integers with $r+s \geq m$. Then there exists a Zariski open neighborhood $\mathcal{O} \subset \mathbf{P}^{1}$ of a k-rational point $0 \in \mathbf{P}^{1}$ and a continuous algebraic map

$$
\Psi=\left(\Psi^{+}, \Psi^{-}\right): \mathcal{C}_{s}(X) \times \mathcal{O} \rightarrow \mathcal{C}_{s}(X)^{2}
$$

satisfying the following properties. Set $\psi_{p}^{ \pm}=\left.\Psi^{ \pm}\right|_{\mathcal{C}_{S}(X) \times\{p\}}$. Then:

(a) For some positive integer $M$ and some $k$-rational point $\mathbf{F}^{*} \in \mathfrak{R}_{X}(d)-\mathfrak{B}(d)_{e}$, chosen as in 1.9, one has

$$
\psi_{0}^{+}=(M+1) \cdot \psi_{\mathbf{F}^{*}}^{+}+M \cdot \psi_{\mathbf{F}^{*}}^{-}, \quad \psi_{0}^{-}=(M+1) \cdot \psi_{\mathbf{F}^{*}}^{-}+M \cdot \psi_{\mathbf{F}^{*}}^{+}
$$

where $\psi_{\mathbf{F}^{*}}^{+}, \psi_{\mathbf{F}^{*}}^{-}: \mathcal{C}_{s}(X) \rightarrow \mathcal{C}_{s}(X)$ are the continuous algebraic maps of Proposition 1.9.a. In particular, for every effective s-cycle $Z$,

$$
Z=\psi_{0}^{+}(Z)-\psi_{0}^{-}(Z)
$$

(b) For any $k$-rational point $p \in \mathcal{O}, \psi_{p}^{ \pm}$determines a pair of linear maps on effective $s$ - cycles

(c) For any effective $s$-cycle $Z$ on $X$, the restriction of $\Psi$ to $\{Z\} \times \mathcal{O}$ determines a rational equivalence $Z_{\psi} \subset \mathbf{P}^{n} \times \mathbf{P}^{1}$ whose fibre above a point $p \in \mathcal{O}$ is $\psi_{p}^{+}(Z)-\psi_{p}^{-}(Z)$

(d) For all effective cycles $Y, Z$ on $X$ of dimensions $r, s$ and degrees $\leq e$ and all $k$ rational points $p \in \mathcal{O}-\{0\}$, any component of excess dimension (i.e., $>r+s-m$ ) of either $|Y| \cap\left|\psi_{p}^{+}(Z)\right|$ or $|Y| \cap\left|\psi_{p}^{-}(Z)\right|$ lies in the singular locus of $X$.

Proof. Choose $\mathbf{F}^{*}$ as in Proposition 1.9 and recall the equality

$$
Z=(-1)^{m+1} R_{\mathbf{F}^{*}}(Z)+\sum_{i=1}^{m}(-1)^{i} \pi_{\mathbf{F}^{i}}^{*}\left\{p_{\mathbf{F}^{i} *}\left\{R_{\mathbf{F}^{i-1}} \circ \cdots \circ R_{\mathbf{F}^{0}}(Z)\right\}\right\} \bullet X
$$


and the fact that $\psi_{\mathbf{F}^{*}}^{ \pm}$are given as the positive and negative parts of the right hand side of (1.9.1). Let $e^{\prime} \geq e$ be a positive integer bounding the degrees of $p_{\mathbf{F}_{*}^{i}}(Y)$ and of $p_{\mathbf{F}_{*}^{i}}\left(R_{\mathbf{F}^{i-1}} \circ \cdots \circ R_{\mathbf{F}^{0}}(Z)\right)$ as $Y$ and $Z$ vary over effective $r$-cycles and $s$-cycles on $X$ (respectively) of degrees $\leq e$, and $i$ varies from 1 to $m$.

We now apply Theorem 2.6 with $e$ replaced by $e^{\prime}$ and with some fixed $c>1$. In fact we apply the theorem twice. We choose two sequences $\mathbf{N}=\left(\mathbf{N}_{1}, \ldots, \mathbf{N}_{E}\right)$ and $\mathbf{N}^{\prime}=$ $\left(\mathbf{N}_{1}^{\prime}, \ldots, \mathbf{N}_{E^{\prime}}^{\prime}\right)$ with $N_{j, i}$ and $N_{j, i}^{\prime}$ sufficiently large as required in Proposition 2.5 , so that

$$
\prod_{j=1}^{E}\left|\mathbf{N}_{j}\right|=M+1 \quad, \quad \prod_{j=1}^{E^{\prime}}\left|\mathbf{N}_{j}^{\prime}\right|=M
$$

for some positive integer $M$. To see that this is possible recall that the integers $N_{j, i}$ can be chosen arbitrarily subject to the inequalities of 2.5. Consequently we may choose the $N_{j, i}$ 's and the $N_{j, i}^{\prime}$ so that $\prod_{j}\left|\mathbf{N}_{j}\right|$ and $\prod_{j}\left|\mathbf{N}_{j}^{\prime}\right|$ are relatively prime. Then there exist positive integers $a$ and $a^{\prime}$ so that $a \prod_{j}\left|\mathbf{N}_{j}\right|-a^{\prime} \prod_{j}\left|\mathbf{N}_{j}^{\prime}\right|= \pm 1$. We may assume, by reordering the choice if necessary, that the difference is +1 , and we then replace $N_{E, t}$ with $a N_{E, t}$ and $N_{E^{\prime}, t}^{\prime}$ with $a^{\prime} N_{E^{\prime}, t}^{\prime}$.

By Proposition 2.5 we obtain sequences $\left(L_{1}, L_{2}, \ldots, L_{E}\right)$ and $\left(L_{1}^{\prime}, L_{2}^{\prime}, \ldots, L_{E^{\prime}}^{\prime}\right)$ of $(t-$ 1)-dimensional linear subspaces, for which the conclusions of 2.5 hold.

We now apply Theorem 2.6 (twice) for the $\left\{\mathbf{N}_{j}\right\},\left\{L_{j}\right\}$ (and the $\left\{\mathbf{N}_{j}^{\prime}\right\},\left\{L_{j}^{\prime}\right\}$ ) chosen above. We fix $k$-rational points $\underline{0}$ and $\underline{0^{\prime}}$ and $k$-rational lines $\ell$ and $\ell^{\prime}$ as in Theorem 2.6, and we let

$$
\Theta_{\underline{\mathbf{N}}}: \mathcal{C}_{s}\left(\mathbf{P}^{m}\right) \times \ell^{\circ} \rightarrow \mathcal{C}_{s}\left(\mathbf{P}^{m}\right) \quad \text { and } \quad \Theta^{\prime} \underline{\mathbf{N}}^{\prime}: \mathcal{C}_{s}\left(\mathbf{P}^{m}\right) \times\left(\ell^{\prime}\right)^{\circ} \rightarrow \mathcal{C}_{s}\left(\mathbf{P}^{m}\right)
$$

be the continuous algebraic maps constructed in 2.6 for this data.

We now choose a dense open subset $\mathcal{O} \subset \mathbf{P}^{1}$ and point $0 \in \mathcal{O}$ together with open immersions $f: \mathcal{O} \hookrightarrow \ell^{\circ}$ and $f^{\prime}: \mathcal{O} \hookrightarrow\left(\ell^{\prime}\right)^{\circ}$ sending 0 to $\underline{0}$ and $\underline{0^{\prime}}$ respectively. We let $\Theta_{\underline{\mathbf{N}}, p}$ denote the restriction of $\Theta_{\underline{\mathbf{N}}}$ to $\mathcal{C}_{s}\left(\mathbf{P}^{m}\right) \times\{f(p)\}$ for any $k$-rational point $p \in \mathcal{O}$. We define $\Psi_{\underline{\mathbf{N}}}$ by

$\Psi_{\underline{\mathbf{N}}}(Z, p)=(-1)^{m+1}(M+1) \cdot R_{\mathbf{F}^{*}}(Z)+\sum_{i=1}^{m}(-1)^{i} \pi_{\mathbf{F}^{i}}^{*}\left\{\Theta_{\underline{\mathbf{N}}, p}\left\{p_{\mathbf{F}^{i} *}\left(R_{\mathbf{F}^{i-1}} \circ \cdots \circ R_{\mathbf{F}^{0}}(Z)\right)\right\}\right\} \bullet X$,

and define $\Psi_{\underline{N}^{\prime}}$ similarly. Finally, we define $\Psi^{ \pm}$to be the positive and negative parts of $\Psi_{\underline{\mathbf{N}}}-\Psi_{\underline{\mathbf{N}}^{\prime}}$, so that

$$
\Psi^{+}(Z, p)-\Psi^{-}(Z, p)=\Psi_{\underline{\mathbf{N}}}(Z, p)-\Psi_{\underline{\mathbf{N}}^{\prime}}(Z, p) .
$$

The fact that $\Psi^{+}, \Psi^{-}$are continuous algebraic maps follows immediately from Proposition 1.9 and Theorem 2.6. Property (a) is part of our definition of $\Psi^{ \pm}$. Property (b) follows immediately from Proposition 1.9 and Theorem 2.6.b. The proof of property (c) is merely a repetition of the proof of Theorem 2.6.c. 
To verify property (d), observe that Theorem 1.7.b implies that any component of excess dimension of $|Y| \cap\left|R_{\mathbf{F}^{*}}(Z)\right|$ lies in the singular locus of $X$. On the other hand, since $p_{\mathbf{F}^{i}}$ is a finite map,

$$
Y, \quad \pi_{\mathbf{F}^{i}}^{*}\left\{\Theta_{\mathbf{N}, p}\left\{p_{\mathbf{F}^{i *}}\left(R_{\mathbf{F}^{i-1}} \circ \cdots \circ R_{\mathbf{F}^{0}}(Z)\right)\right\}\right\} \bullet X
$$

intersect properly for any $i, 1 \leq i \leq m$, if and only if

$$
p_{\mathbf{F}^{i *}}(Y), \quad \Theta_{\mathbf{N}, p}\left\{p_{\mathbf{F}^{i} *}\left(R_{\mathbf{F}^{i-1}} \circ \cdots \circ R_{\mathbf{F}^{0}}(Z)\right)\right\}
$$

intersect properly. These do intersect properly whenever $Y, Z$ are effective cycles of degrees $\leq e$ and $p \in \mathcal{O}-\{0\}$ by Theorem 2.6.d. The analogous remarks apply with $\mathbf{N}$ replaced by $\mathbf{N}^{\prime}$. This completes the proof.

Remark 3.2. The proof of Theorem 3.1 in fact proves the more general result that one can "move" effective $s$-cycles $Z$ of degrees $\leq e$ to meet properly (off the singular locus of $X$ ) all effective cycles $Y$ of dimension $\geq m-s$ and degree $\leq e$. Namely, we verify that the arguments of section 1 apply when $\mathcal{C}_{r, \leq e}(X)$ is replaced by

$$
\mathcal{C}_{\geq n-s, \leq e}(X) \equiv \coprod_{r \geq m-s, d \leq e} \mathcal{C}_{r, d}(X)
$$

Similarly, the arguments of section 2 apply when we replace $\mathcal{C}_{r, \leq e}\left(\mathbf{P}^{n}\right)$ by $\mathcal{C}_{\geq s, \leq e}\left(\mathbf{P}^{n}\right)$ and $t=n-r$ by $s$.

When $k=\mathbf{C}$ our Main Theorem can be phrased differently and in a way that may appeal to complex geometers. In this case the $p$-cycles form a topological abelian group where the topology is compactly generated by the images of $\mathcal{C}_{p, \leq e}(X) \times \mathcal{C}_{p, \leq e}(X)$ under the natural projection

$$
\pi: \mathcal{C}_{p}(X) \times \mathcal{C}_{p}(X) \longrightarrow \mathcal{Z}_{p}(X)
$$

We set $\mathcal{Z}_{p, \leq e}(X) \stackrel{\text { def }}{=} \pi\left\{\mathcal{C}_{p, \leq e}(X) \times \mathcal{C}_{p, \leq e}(X)\right\}$

Corollary 3.3. Let $X \subset P_{\mathbf{C}}^{n}$ be a complex projective variety of dimension $m$. Let $r, s, e$ be non-negative integers with $r+s \geq m$. Then there exist a Zariski open neighborhood $\mathcal{O}$ of $\{0\}$ in $\mathbf{C}$ and a continuous algebraic map

$$
\tilde{\Psi}: \mathcal{C}_{s}(X) \times \mathcal{O} \longrightarrow \mathcal{C}_{s}(X)^{2}
$$

such that $\pi \circ \Psi$ induces by linearity a continuous map

$$
\Psi: \mathcal{Z}_{s}(X) \times \mathcal{O} \longrightarrow \mathcal{Z}_{s}(X)
$$

satisfying the following properties. Set $\psi_{p}=\left.\Psi\right|_{\mathcal{Z}_{s}(X) \times\{p\}}$ for $p \in \mathcal{O}$.

(a) $\psi_{0}=$ Id. 
(b) For any $Z \in \mathcal{Z}_{s}(X)$ and any $p \neq 0$ in $\mathcal{O}$, the restriction

$$
\left.\Psi\right|_{\{Z\} \times \mathcal{O}}:\{Z\} \times \mathcal{O} \longrightarrow \mathcal{Z}_{s}(X)
$$

determines a rational equivalence between $Z$ and $\psi_{p}(Z)$.

(c) For any $p \in \mathcal{O}, \psi_{p}$ is a continuous group homomorphism.

(d) For any $Z \in \mathcal{Z}_{s, \leq e}(X), Y \in$ and any $p \neq 0$ in $\mathcal{O}$, each component of excess dimension (i.e., $>r+s-m$ ) of the intersection

$$
|Y| \cap\left|\psi_{p}(Z)\right|
$$

is contained in the singular locus of $X$.

Remark. We may paraphrase Corollary 3.3 in terms of families of cycles. Let us say that a collection $\left\{Y_{\alpha} ; \alpha \in A\right\}$ of $r$-cycles on $X$ is a family of cycles of bounded degree if there exists some positive integer $e$ and some function $g: A \rightarrow \mathcal{Z}_{r, \leq e}(X)$ such that $Y_{\alpha}=g(\alpha)$ for all $\alpha \in A$. Then Corollary 3.3 asserts that for a given family $\left\{Y_{\alpha} ; \alpha \in A\right\}$ of $r$-cycles of bounded degree and a given family $\left\{Z_{\beta} ; \beta \in B\right\}$ of $s$-cycles of bounded degree, $\Psi$ provides a continuous move of all $s$-cycles on $X$ with the following property: for any $\alpha \in A, \beta \in B, p \in \mathcal{O}-\{0\}$ each component of $\left|Y_{\alpha}\right| \cap\left|\psi_{p}\left(Z_{\beta}\right)\right|$ of excess intersection lies in the singular locus of $X$.

Moreover, Corollary 3.3 further asserts how such a move is algebraic, thereby moving one "algebraic family" to another.

As demonstrated in [R], [S], any s-cycle $Z$ can be moved to intersect properly any $r$ cycle $Y$ on a smooth, $m$-dimensional variety $X$ with $r+s \geq m$. This does not immediately imply that the intersection product is well defined, for one must verify that if $Z^{\prime}, Z^{\prime \prime}$ are both obtained from $Z$ by moving $Z$ and both intersect $Y$ properly, then $Y \bullet Z^{\prime}, Y \bullet Z^{\prime \prime}$ are rationally equivalent. As we see in the proof of the following theorem, the fact that such an intersection product is well defined is a straight-forward consequence of Theorem 3.1

Theorem 3.4 Let $X$ be a smooth, quasi-projective variety of dimension $m$ and let $Y, Z$ be cycles on $X$ of dimension $r, s$ with $r+s \geq m$. If both $Z^{\prime}$ and $Z^{\prime \prime}$ are rationally equivalent to $Z$ and intersect $Y$ properly, then $Y \bullet Z^{\prime}, Y \bullet Z^{\prime \prime}$ are also rationally equivalent.

Proof. We first consider the case in which $k$ is either an infinite field or a "sufficiently large" finite field as in the statement of Theorem 3.1. We may assume that the rational equivalences relating $Z$ to $Z^{\prime}$ and $Z^{\prime \prime}$ are given by irreducible, smooth, rational curves. Thus, setting $T=\operatorname{Spec} k[x, y] / x y$, there is a (flat) family $Z_{T}=\left\{Z_{t}: t \in T\right\}$ on $T \times X$ of cycles on $X$ parametrized by $T$ giving a rational equivalence between $Z=Z_{0,0}, Z^{\prime}=Z_{1,0}$, and $Z^{\prime \prime}=Z_{0,1}$. Choose some projective closure $\bar{X}$ of $X$.

Write $Z_{T}=Z_{T}^{+}-Z_{T}^{-}$, where $Z_{T}^{+}, Z_{T}^{-}$are flat families of effective cycles over $T$. Let $B_{i}, i=1,2$ denote the two branches of $T$ and let $Z_{B_{i}}^{+}$(respectively, $Z_{B_{i}}^{-}$) denote the restriction of $Z_{T}^{+}$(resp., $Z_{T}^{-}$) to $B_{i}$. Then the closures $\bar{Z}_{B_{i}}^{ \pm}$in $B_{i} \times \bar{X}$ of $Z_{B_{i}}^{ \pm}$are flat over 
$B_{i}$. Let $\bar{Z}_{i}^{ \pm}$denote the fibres of $\bar{Z}_{B_{i}}^{ \pm}$above $(0,0)$, and let $\bar{Z}_{0}^{ \pm}$denote the closures in $\bar{X}$ of the fibres of $Z_{T}^{ \pm}$above $(0,0)$. Then

$$
\bar{Z}_{i}^{+}=\bar{Z}_{0}^{+}+A_{i}^{+}, \bar{Z}_{i}^{-}=\bar{Z}_{0}^{-}+A_{i}^{-}
$$

where $A_{1}^{+}, A_{1}^{-}, A_{2}^{+}, A_{2}^{-}$are effective cycles on $\bar{X}$ supported on $\bar{X}-X$.

We define

$$
\hat{Z}_{T}^{+}=\left(\bar{Z}_{B_{1}}^{+}+\left(A_{2}^{+} \times T\right)\right)+\left(\bar{Z}_{B_{2}}^{+}+\left(A_{1}^{+} \times T\right)\right)
$$

and observe that $\hat{Z}_{T}^{+}$determines a continuous algebraic map $\varphi^{+}: T \rightarrow \mathcal{C}_{s}(\bar{X})$ because the restrictions to $B_{1}, B_{2}$ are flat and their futher restrictions to $(0,0)$ are equal. We similarly define $\hat{Z}_{T}^{-}$determining $\varphi^{-}: T \rightarrow \mathcal{C}_{s}(\bar{X})$, and set

$$
\varphi=\left(\varphi^{+}, \varphi^{-}\right): T \rightarrow \mathcal{C}_{s}(\bar{X})^{2}
$$

We now apply Theorem 3.1 with $e$ greater than the degrees of some closure of the positive and negative parts of $Y$ as well as the degrees of $\hat{Z}_{t}^{+}, \hat{Z}_{t}^{-}$for all $t \in T$ to obtain

$$
\tilde{\Psi} \circ(\varphi \times 1): T \times \ell \longrightarrow \mathcal{C}_{s}(\bar{X})^{2}
$$

for some and some $k$ rational line $\ell$. Then for all $k$-rational points $p \in \ell-\{0\}$, the restriction

$$
\tilde{\psi}_{R}: R \longrightarrow \mathcal{C}_{s}(\bar{X})^{2}
$$

of $\tilde{\Psi} \circ(\varphi \times 1)$ to

$$
R \equiv(\{(1,0)\} \times \ell) \cup(T \times\{p\}) \cup(\{(0,1)\} \times \ell)
$$

provides a rational equivalence between $\left(\hat{Z}_{T}\right)_{1,0}$ and $\left(\hat{Z}_{T}\right)_{0,1}$ through cycles each of which meet $Y$ properly when restricted to $X$. Thus, sending $t \in R$ to $\left.\left.Y \bullet\left(\tilde{\psi}_{R}(t)\right)\right|_{X}\right)$ determines a rational equivalence between $Y \bullet Z^{\prime}, Y \bullet Z^{\prime \prime}$.

Suppose now that $k$ is an arbitary finite field and $k \rightarrow k^{\prime}$ is a finite field extension with $k^{\prime}$ sufficiently large. Let $\pi: X_{k^{\prime}} \rightarrow X$ denote the finite, flat map given by base extension, where $X_{k^{\prime}} \equiv X \times_{\operatorname{Spec}(k)} \operatorname{Spec}\left(k^{\prime}\right)$; this is a map of algebraic $k$ schemes, but not a map of quasi-projective algebraic varieties over $k$. By the preceding argument, the rational equivalence class of $\pi^{*}\left(Y \bullet Z^{\prime}-Y \bullet Z^{\prime \prime}\right)$ is 0 in the Chow group of $r+s-m$ cycles on $X_{k^{\prime}}$. Since $\pi_{*} \circ \pi^{*}$ equals multiplication by $d^{\prime}=\left[k^{\prime}: k\right]$ on cycles, $\pi_{*} \circ \pi^{*}$ also equals multiplication by $d^{\prime}$ on rational equivalence classes of cycles. By considering $\pi^{*}\left(Y \bullet Z^{\prime}-Y \bullet Z^{\prime \prime}\right)$ for two such such sufficiently large field extensions $k \rightarrow k^{\prime}, k \rightarrow k^{\prime \prime}$ of relatively prime degrees $d^{\prime}, d^{\prime \prime}$ over $k$, we conclude that $Y \bullet Z^{\prime}, Y \bullet Z^{\prime \prime}$ are rationally equivalent on $X$.

In $[\mathrm{F}-\mathrm{G}]$, an intersection product was defined on the Lawson homology groups of a smooth, complex, quasi-projective variety $X$ of dimension $m$ :

$$
L_{r} H_{2 r+i}(X) \otimes L_{s} H_{2 r+j}(X) \longrightarrow L_{r+s-m} H_{2(r+s-m)+i+j}(X), \quad r+s \geq m .
$$


This product was defined using an argument involving the Fulton-MacPherson "Deformation to the normal cone" technique together with a homotopy lifing argument. The following consequence of Theorem 3.1 gives a more concrete construction of this intersection product for $X$ projective as well as smooth. Even in this case, the constructon of [F-G] remains useful in establishing the numerous good properties of this product.

Theorem 3.5 Let $X$ be a smooth, complex projective variety of dimension $m$ and let $r, s$ be non-negative integers with $r+s \geq m$. Let $\mathcal{Z}_{r}(X)$ denote the topological abelian group of $r$-cycles on $X$ obtained as the "naive" group completion of the abelian monoid $\mathcal{C}_{s}(X)$ provided with the analytic topology. Then the pairing on homotopy groups induced by the intersection product of [F-G]

$$
\pi_{i}\left(\mathcal{Z}_{r}(X)\right) \otimes \pi_{j}\left(\mathcal{Z}_{s}(X)\right) \stackrel{\bullet}{\longrightarrow} \pi_{i+j}\left(\mathcal{Z}_{r+s-m}(X)\right)
$$

can be represented as follows: given homotopy classes $\alpha \in \pi_{i}\left(\mathcal{Z}_{r}(X)\right), \beta \in \pi_{j}\left(\mathcal{Z}_{s}(X)\right)$, there exist representative (base point preserving) maps $a: S^{i} \rightarrow \mathcal{Z}_{r}(X), \quad b: S^{j} \rightarrow \mathcal{Z}_{s}(X)$ such that $a(t), b(u)$ intersect properly for all $t \in S^{i}, u \in S^{j}$ and such that the map sending $t \wedge u$ to the intersection of $a(t)$ and $b(u)$ is a continuous map $a \cdot b: S^{i+j} \rightarrow \mathcal{Z}_{r+s-m}(X)$ which represents $\alpha \bullet \beta$.

Proof. Since the topology on $\mathcal{Z}_{k}(X)$ is compactly generated by the images of $\mathcal{C}_{k, \leq e}(X)^{2}$ for $e>0$, we may choose $e$ sufficiently large that arbitrary representatives $a, b^{\prime}$ of $\alpha, \beta$ lift to continuous maps $\tilde{a}: \tilde{S}^{i} \rightarrow \mathcal{C}_{r, \leq e}(X)^{2}, \tilde{b}^{\prime}: \tilde{S}^{j} \rightarrow \mathcal{C}_{s, \leq e}(X)^{2}$.

The continuous algebraic map $\Psi$ of Theorem 3.1 chosen for this $e$ determines a continuous map (for the analytic topology)

$$
\Psi: \mathcal{Z}_{s}(X) \times \mathcal{O} \rightarrow \mathcal{Z}_{s}(X)
$$

with the property that $\psi_{0}$ (i.e., the restriction of $\Psi$ to $\left.\mathcal{Z}_{s}(X) \times\{0\}\right)$ is the identity. Define

$$
b=\Psi \circ\left(b^{\prime} \times i_{p}\right): S^{j} \rightarrow \mathcal{Z}_{s}(X) \times \mathcal{O} \rightarrow \mathcal{Z}_{s}(X)
$$

for some $p \in \mathcal{O}-\{0\}$, where $i_{p}: S^{j} \rightarrow \mathcal{O}$ is the constant map with value $p$. By Theorem 3.1.d and our choice of $e$, the $r$-cycles $a(t), b(u)$ intersect properly for all $t \in S^{i}, u \in S^{j}$.

The intersection of cycles meeting properly is continuous, so that $a, b$ determine a continuous map $a \cdot b: S^{i+j} \rightarrow \mathcal{Z}_{r+s-m}(X)$. The fact that this map represents $\alpha \bullet \beta$ follows from $[\mathrm{F}-\mathrm{G} ; 3.5 . \mathrm{a}]$.

Remark 3.6. Our techniques fail to provide a representation of the intersection product on Lawson homology group of complex, smooth varieties $X$ which are quasi-projective but not projective. This is because the "moves" we construct for $s$-cycles on a smooth completion $\bar{X}$ of $X$ do not restrict to moves on $s$ - cycles on $\bar{X}-X$.

We finish with a theorem that captures the fundamental assertion of the duality theory in [F-L2]. It says that on a flat family of varieties, any cycle (of dimension greater than 
that of the base) can be moved so that it becomes itself a family of cycles in this family of varieties. The assertion holds in fact for any algebraic collection of cycles on the family.

Theorem 3.7 Let $X$ be a smooth projective variety, $f: X \rightarrow B$ a flat morphism of varieties over $k$, and $s$ an integer $\geq \operatorname{dim}(X)-\operatorname{dim}(B)$. Assume that $k$ is an infinite field. Then for any positive integer $e$, there exist a Zariski open neighbrohood $\mathcal{O} \subset \mathbf{P}^{1}$ of a $k$-rational point $0 \in \mathbf{P}^{1}$ and continuous algebraic maps

$$
\Psi=\left(\Psi^{+}, \Psi^{-}\right): \mathcal{C}_{s}(X) \times \mathcal{O} \rightarrow \mathcal{C}_{s}(X)^{2}
$$

satisfying the following properties (where $\psi_{p}^{ \pm}$denotes the restriction of $\Psi^{ \pm}$to $\mathcal{C}_{s}(X) \times\{p\}$ ):

(a) $Z=\psi_{0}^{+}(Z)-\psi_{0}^{-}(Z)$ for every effective s-cycle $Z$ on $X$.

(b) For any effective $s$-cycle $Z$, the restriction of $\Psi$ to $\{Z\} \times \mathcal{O}$ determines a rational equivalence $Z_{\mathcal{O}} \subset X \times \mathbf{P}^{1}$ whose fibre equals $\psi_{p}^{+}(Z)-\psi_{p}^{-}(Z)$.

(c) For every effective s-cycle $Z$ of degree $\leq e$, the cycles $\psi_{p}^{+}(Z), \psi_{p}^{-}(Z)$ intersect properly each fibre $f^{-1}(b), b \in B$ of $f$ whenever $p$ is a rational point of $\mathcal{O}-\{0\}$.

Proof. Apply Theorem 3.1 with $e$ replaced by the larger of the integer $e$ of the statement of this theorem and the maximum of the degrees of the fibres $f^{-1}(b), b \in B$ of $f$. 


\section{References}

[Chev] C. Chevalley, Anneaux de Chow et Applications, Séminaire Chevalley, Secrétariant Math., Pairs (1958).

[Chow] W.S. Chow, On the equivalence classes of cycles in an algebraic variety, Annals of Math. 64 (1956), 450-479.

[F] E. Friedlander, Algebraic cocycles, Chow varieties, and Lawson homology, Compositio Math. 77 (1991), 55-93.

[F-G] E. Friedlander and O. Gabber, Cycles spaces and intersection theory, in Topological Methods in Modern Mathematics (1993), 325-370.

[F-L] E. Friedlander and H.B. Lawson, A theory of algebraic cocycles, Annals of Math. 136 (1992), 361-428.

[F-L2] E. Friedlander and H.B. Lawson, Duality relating cycle and cocyle spaces. In preparation.

[F-V] E. Friedlander and V. Voevodsky. Bivariant cycle cohomology. Preprint.

[Fu] W. Fulton, Intersection Theory, Springer-Verlag, New York, 1984.

[L] H.B. Lawson, Algebraic cycles and homotopy theory, Annals of Math. 129 (1989), 253-291.

[L-F] P. Lima-Filho, Completions and fibrations for topological monoids and excision for Lawson homology, Trans. A.M.S. 340 (1993), no. 1, 127-147.

[Mat] H. Matsumura, Commutative Algebra, Benjamin, 1970.

[R] J. Roberts, Chow's Moving Lemma, in Algebraic Geometry Oslo 1970 (1972), 89-96.

[S1] P. Samuel, Méthodes d'algèbre abstraite en géométrie algébrique, Ergebnisse der math., Springer- Verlag, 1955.

[S2] P. Samuel, Rational equivalence of arbitrary cycles, Amer. J. Math. 78 (1956), 383400. 